\title{
Existence, uniqueness, and regularity for stochastic evolution equations with irregular initial values
}

\author{
Adam Andersson, Arnulf Jentzen, and Ryan Kurniawan
}

August 7, 2018

\begin{abstract}
In this article we develop a framework for studying parabolic semilinear stochastic evolution equations (SEEs) with singularities in the initial condition and singularities at the initial time of the time-dependent coefficients of the considered SEE. We use this framework to establish existence, uniqueness, and regularity results for mild solutions of parabolic semilinear SEEs with singularities at the initial time. We also provide several counterexample SEEs that illustrate the optimality of our results.
\end{abstract}

\section{Contents}

1 Introduction 1

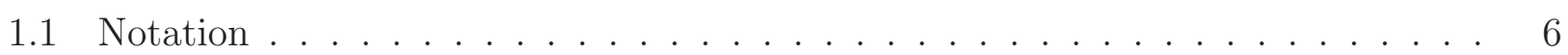

2 Stochastic evolution equations (SEEs) with singularities at the initial time 7

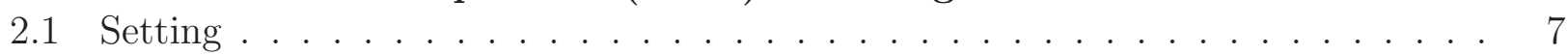

2.2 Predictable stochastic processes with singularities at the initial time . . . . . 7

2.3 A perturbation estimate for stochastic processes . . . . . . . . . . . . 14

2.4 Existence, uniqueness, and regularity for SEEs with singularities at the initial

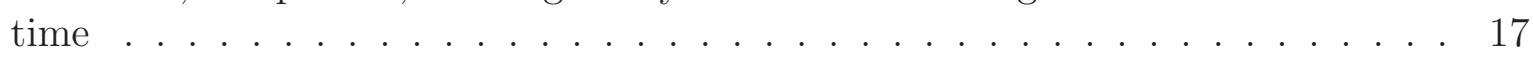

3 Examples and counterexamples for SEEs with irregular initial values 23

3.1 Setting . . . . . . . . . . . . . . . . . . . . . . . . . . . . . . . . . . .

3.2 Stochastic heat equations with linear multiplicative noise . . . . . . . . . . 23

3.3 Stochastic heat equations with nonlinear multiplicative noise . . . . . . . . . 28

3.4 Nonlinear heat equations . . . . . . . . . . . . . . . . . . . 31

\section{Introduction}

There are a number of existence, uniqueness, and regularity results for mild solutions of semilinear stochastic evolution equations (SEEs) in the literature; see, e.g., [10, 11, 4, 28, 16, 18, 21, 27. 
and the references mentioned therein. In this work we extend the above cited results by adding singularities in the initial condition and by introducing singularities at the initial time of the time-dependent coefficients of the considered SEE; cf., e.g., Chen \& Dalang [7, 8]. To be more specific, in the first main result of this work (see Proposition 2.7 below) we establish a general perturbation estimate (see (5) in this introductory section below) for a general class of stochastic processes which allows us to derive a priori bounds (see, e.g., (7) in this introductory section below) for solutions and numerical approximations of SEEs with singularities at the initial time. This perturbation estimate, in turn, is used to prove the second main result of this article (see Theorem 2.9 below) which establishes existence, uniqueness, and regularity properties for solutions of SEEs with singularities at the initial time (see (6) and (7) in this introductory section below). As an application of our perturbation estimate and this second main result of our article, we reveal a regularity barrier (see (10) in this introductory section below) for the initial condition of SEEs under which the considered SEE has unique solutions which are Lipschitz continuous with respect to initial values (see Corollary 2.10 below). By means of several counterexamples (see Proposition 3.2. Proposition 3.4, and Proposition 3.5 below) we also demonstrate that this regularity barrier can in general not essentially be improved (cf. (12) and (13) in this introductory section below). We illustrate the above findings in the case of possibly nonlinear stochastic heat equations on an interval such as the continuous version of the parabolic Anderson model on an interval (cf. Corollary 3.1, Proposition 3.2, and Proposition 3.3 below). Existence, uniqueness, and regularity results for possibly nonlinear stochastic heat equations on the whole real line with rough initial values, that is, signed Borel measures with exponentially growing tails over $\mathbb{R}$ as initial values can be found in Chen \& Dalang [7, 8] (see Theorem 2.4 in Chen \& Dalang [8] for an existence and uniqueness result and a priori estimates and see Theorem 3.1 in Chen \& Dalang [7] for a Hölder regularity result). Moreover, Proposition 2.11 in Chen \& Dalang [8] disproves the existence of a solution of the considered stochastic heat equation in the case of a specific rough initial value, that is, the derivative of the Dirac delta measure at zero as the initial value.

To illustrate the results of this article in more details, we assume the following setting throughout this introductory section. Let $\left(H,\|\cdot\|_{H},\langle\cdot, \cdot\rangle_{H}\right)$ and $\left(U,\|\cdot\|_{U},\langle\cdot, \cdot\rangle_{U}\right)$ be nontrivial separable $\mathbb{R}$-Hilbert spaces, let $T \in(0, \infty), \eta \in \mathbb{R}, p \in[2, \infty), \alpha \in[0,1), \hat{\alpha} \in(-\infty, 1)$, $\beta \in[0,1 / 2), \hat{\beta} \in(-\infty, 1 / 2), L_{0}, \hat{L}_{0}, L_{1}, \hat{L}_{1} \in[0, \infty), \kappa=\mathbb{1}_{(0, \infty)}\left(L_{1}\right)$ satisfy $\kappa(\alpha+\hat{\alpha})<3 / 2$, let $\left(\Omega, \mathcal{F}, \mathbb{P},\left(\mathcal{F}_{t}\right)_{t \in[0, T]}\right)$ be a stochastic basis, let $\left(W_{t}\right)_{t \in[0, T]}$ be an $\operatorname{Id}_{U}$-cylindrical $\left(\mathcal{F}_{t}\right)_{t \in[0, T]}$-Wiener process, let $A: D(A) \subseteq H \rightarrow H$ be a generator of a strongly continuous analytic semigroup with $\operatorname{spectrum}(A) \subseteq\{z \in \mathbb{C}: \operatorname{Re}(z)<\eta\}$, let $\left(H_{r},\|\cdot\|_{H_{r}},\langle\cdot, \cdot\rangle_{H_{r}}\right), r \in \mathbb{R}$, be a family of interpolation spaces associated to $\eta-A$ (cf., e.g., [26, Section 3.7]), let $\mathbf{F}:[0, T] \times \Omega \times H \rightarrow H_{-\alpha}$ be a $\left(\operatorname{Pred}\left(\left(\mathcal{F}_{t}\right)_{t \in[0, T]}\right) \otimes \mathcal{B}(H)\right) / \mathcal{B}\left(H_{-\alpha}\right)$-measurable mapping, let $\mathbf{B}:[0, T] \times \Omega \times H \rightarrow H S\left(U, H_{-\beta}\right)$ be a $\left(\operatorname{Pred}\left(\left(\mathcal{F}_{t}\right)_{t \in[0, T]}\right) \otimes \mathcal{B}(H)\right) / \mathcal{B}\left(H S\left(U, H_{-\beta}\right)\right)$-measurable mapping, assume for all $t \in(0, T]$, $X, Y \in \mathcal{L}^{p}(\mathbb{P} ; H)$ that

$$
\begin{gathered}
\|\mathbf{F}(t, X)-\mathbf{F}(t, Y)\|_{L^{p}\left(\mathbb{P} ; H_{-\alpha}\right)} \leq L_{0}\|X-Y\|_{L^{p}(\mathbb{P} ; H)},\|\mathbf{F}(t, 0)\|_{L^{p}\left(\mathbb{P} ; H_{-\alpha}\right)} \leq \hat{L}_{0} t^{-\hat{\alpha}} \\
\|\mathbf{B}(t, X)-\mathbf{B}(t, Y)\|_{L^{p}\left(\mathbb{P} ; H S\left(U, H_{-\beta}\right)\right)} \leq L_{1}\|X-Y\|_{L^{p}(\mathbb{P} ; H)},\|\mathbf{B}(t, 0)\|_{L^{p}\left(\mathbb{P} ; H S\left(U, H_{-\beta}\right)\right)} \leq \hat{L}_{1} t^{-\hat{\beta}}
\end{gathered}
$$

for every $a, b \in(-\infty, 1)$ let $\mathrm{E}_{a, b}:[0, \infty) \rightarrow[0, \infty)$ be the function which satisfies for all $x \in$ $[0, \infty)$ that $\mathrm{E}_{a, b}[x]=1+\sum_{n=1}^{\infty} x^{n} \prod_{k=0}^{n-1} \int_{0}^{1} t^{-b}(1-t)^{k(1-b)-a} d t$ (cf., e.g., [14, Chapter 7]), for 
every $r \in[0,1]$ let $\chi_{r} \in(0, \infty)$ be the real number given by $\chi_{r}=\sup _{t \in(0, T]} t^{r}\left\|(\eta-A)^{r} e^{t A}\right\|_{L(H)}$ (see, e.g., [24, Lemma 11.36]), and for every $\lambda \in\left(-\infty, \frac{1}{2}\left[1+\mathbb{1}_{\{0\}}\left(L_{1}\right)\right]\right)$ let $\Theta_{\lambda} \in[0, \infty)$ be the real number given by

$$
\Theta_{\lambda}=2^{\kappa / 2}\left|\mathrm{E}_{(1+\kappa) \lambda, \max \{\alpha, 2 \beta \kappa\}}\left[\left|\frac{\chi_{\alpha} L_{0} 2^{\kappa / 2} T^{(1-\alpha)}}{(1-\alpha)^{\kappa / 2}}+\chi_{\beta} L_{1} \sqrt{p(p-1) T^{(1-2 \beta)}}\right|^{(1+\kappa)}\right]\right|^{(2-\kappa) / 2} .
$$

In displays (5)-(13) below we illustrate the above framework through several examples and applications.

Our first result is a suitable perturbation estimate for predictable stochastic processes. More formally, in Proposition 2.7 below we prove for all $\delta \in \mathbb{R}, \lambda \in\left(-\infty, \frac{1}{2}\left[1+\mathbb{1}_{\{0\}}\left(L_{1}\right)\right]\right)$ and all $\left(\mathcal{F}_{t}\right)_{t \in[0, T]}$-predictable stochastic processes $Y^{1}, Y^{2}:[0, T] \times \Omega \rightarrow H_{\delta}$ with

$$
\cup_{k \in\{1,2\}} Y^{k}((0, T] \times \Omega) \subseteq H \quad \text { and } \quad \limsup _{r \nearrow \frac{1}{2}\left[1+\mathbb{1}_{\{0\}}\left(L_{1}\right)\right]} \max _{k \in\{1,2\}} \sup _{t \in(0, T]} t^{r}\left\|Y_{t}^{k}\right\|_{L^{p}(\mathbb{P} ; H)}<\infty
$$

that $\forall t \in[0, T]: \mathbb{P}\left(\sum_{k=1}^{2} \int_{0}^{t}\left\|e^{(t-s) A} \mathbf{F}\left(s, Y_{s}^{k}\right)\right\|_{H}+\left\|e^{(t-s) A} \mathbf{B}\left(s, Y_{s}^{k}\right)\right\|_{H S(U, H)}^{2} \mathrm{~d} s<\infty\right)=1$ and

$$
\begin{array}{r}
\sup _{t \in(0, T]}\left[t^{\lambda}\left\|Y_{t}^{1}-Y_{t}^{2}\right\|_{L^{p}(\mathbb{P} ; H)}\right] \leq \sup _{t \in(0, T]}\left[t^{\lambda} \| Y_{t}^{1}-\int_{0}^{t} e^{(t-s) A} \mathbf{F}\left(s, Y_{s}^{1}\right) \mathrm{d} s-\int_{0}^{t} e^{(t-s) A} \mathbf{B}\left(s, Y_{s}^{1}\right) \mathrm{d} W_{s}\right. \\
\left.+\int_{0}^{t} e^{(t-s) A} \mathbf{F}\left(s, Y_{s}^{2}\right) \mathrm{d} s+\int_{0}^{t} e^{(t-s) A} \mathbf{B}\left(s, Y_{s}^{2}\right) \mathrm{d} W_{s}-Y_{t}^{2} \|_{L^{p}(\mathbb{P} ; H)}\right] \Theta_{\lambda} .
\end{array}
$$

We note that the right hand side of (5) might be infinite. Moreover, we would like to emphasize that $Y^{1}$ and $Y^{2}$ in (5) are arbitrary $\left(\mathcal{F}_{t}\right)_{t \in[0, T]}$-predictable stochastic processes which satisfy (44) and, in particular, we emphasize that $Y^{1}$ and $Y^{2}$ do not need to be solution processes of some SEEs. Estimate (5) follows from an appropriate application of a generalized Gronwall-type inequality (see the proof of Proposition 2.7 below for details).

We use inequality (5) to establish an existence, uniqueness, and regularity result for SEEs with singularities at the initial time. More precisely, in Theorem 2.9 below we prove that for all $\delta \in\left(-\infty, \frac{1}{2}\left[1+\mathbb{1}_{\{0\}}\left(L_{1}\right)\right]\right), \lambda \in\left[\max \{\delta, \alpha+\hat{\alpha}-1, \beta+\hat{\beta}-1 / 2\}, \frac{1}{2}\left[1+\mathbb{1}_{\{0\}}\left(L_{1}\right)\right]\right)$, $\xi \in \mathcal{L}^{p}\left(\left.\mathbb{P}\right|_{\mathcal{F}_{0}} ; H_{-\max \{\delta, 0\}}\right)$ with $\sup _{t \in(0, T]} t^{\delta}\left\|e^{t A} \xi\right\|_{L^{p}(\mathbb{P} ; H)}<\infty$ it holds (i) that there exists an upto-modifications unique $\left(\mathcal{F}_{t}\right)_{t \in[0, T]}$-predictable stochastic process $X:[0, T] \times \Omega \rightarrow H_{-\max \{\delta, 0\}}$ which satisfies for all $t \in[0, T]$ that $X((0, T] \times \Omega) \subseteq H$, that $\sup _{s \in(0, T]} s^{\lambda}\left\|X_{s}\right\|_{L^{p}(\mathbb{P} ; H)}<\infty$, that $\mathbb{P}\left(\int_{0}^{t}\left\|e^{(t-s) A} \mathbf{F}\left(s, X_{s}\right)\right\|_{H}+\left\|e^{(t-s) A} \mathbf{B}\left(s, X_{s}\right)\right\|_{H S(U, H)}^{2} \mathrm{~d} s<\infty\right)=1$, and $\mathbb{P}$-a.s. that

$$
X_{t}=e^{t A} \xi+\int_{0}^{t} e^{(t-s) A} \mathbf{F}\left(s, X_{s}\right) \mathrm{d} s+\int_{0}^{t} e^{(t-s) A} \mathbf{B}\left(s, X_{s}\right) \mathrm{d} W_{s}
$$

and (ii) that

$$
\begin{aligned}
& \sup _{t \in(0, T]}\left[t^{\lambda}\left\|X_{t}\right\|_{L^{p}(\mathbb{P} ; H)}\right] \leq T^{\lambda} \Theta_{\lambda} \\
& \quad \cdot\left[\frac{\sup _{t \in(0, T]}\left(t^{\delta}\left\|e^{t A} \xi\right\|_{L^{p}(\mathbb{P} ; H)}\right)}{T^{\delta}}+\frac{\chi_{\alpha} \hat{L}_{0} \mathbb{B}(1-\alpha, 1-\hat{\alpha})}{T^{(\alpha+\hat{\alpha}-1)}}+\frac{\chi_{\beta} \hat{L}_{1}|p(p-1) \mathbb{B}(1-2 \beta, 1-2 \hat{\beta})|^{1 / 2}}{\sqrt{2} T^{(\beta+\hat{\beta}-1 / 2)}}\right]<\infty .
\end{aligned}
$$


We would like to point out that inequality (7) under the generality of (11) and (2) is a crucial ingredient to establish essentially sharp weak convergence rates for numerical approximations of SEEs with possibly smooth initial values (see the last paragraph in this introductory section for more details).

Inequality (17) follows from the perturbation estimate (15) (with $Y^{1}=X$ and $Y^{2}=0$ in the notation of (5)). We now illustrate Theorem 2.9 and (6) (7), respectively, by some examples. In particular, in Corollary 2.10 below we prove by an application of Theorem 2.9 that for all $F \in \operatorname{Lip}\left(H, H_{-\alpha}\right), B \in \operatorname{Lip}\left(H, H S\left(U, H_{-\beta}\right)\right), \hat{\delta}=\frac{1}{2}\left[1+\mathbb{1}_{\{0\}}\left(|B|_{\operatorname{Lip}\left(H, H S\left(U, H_{-\beta}\right)\right)}\right)\right]$ it holds (i) that there exist up-to-modifications unique $\left(\mathcal{F}_{t}\right)_{t \in[0, T]}$-predictable stochastic processes $X^{x}:[0, T] \times$ $\Omega \rightarrow H_{-\delta}, x \in H_{-\delta}, \delta \in[0, \hat{\delta})$, which fulfill for all $q \in[2, \infty), \delta \in[0, \hat{\delta}), x \in H_{-\delta}, t \in[0, T]$ that $X^{x}((0, T] \times \Omega) \subseteq H$, that $\sup _{s \in(0, T]} s^{\delta}\left\|X_{s}^{x}\right\|_{L^{q}(\mathbb{P} ; H)}<\infty$, and $\mathbb{P}$-a.s. that

$$
X_{t}^{x}=e^{t A} x+\int_{0}^{t} e^{(t-s) A} F\left(X_{s}^{x}\right) \mathrm{d} s+\int_{0}^{t} e^{(t-s) A} B\left(X_{s}^{x}\right) \mathrm{d} W_{s}
$$

and (ii) that

$$
\forall \delta \in[0, \hat{\delta}), q \in[2, \infty): \sup _{\substack{x, y \in H_{-\delta}, t \in(0, T] \\ x \neq y}} \sup _{\substack{ \\\max }} \max \left\{\frac{t^{\delta}\left\|X_{t}^{x}\right\|_{L^{q(\mathbb{P} ; H)}}}{\max \left\{1,\|x\|_{H_{-}}\right\}}, \frac{t^{\delta}\left\|X_{t}^{x}-X_{t}^{y}\right\|_{L^{q}(\mathbb{P} ; H)}}{\|x-y\|_{H_{-\delta}}}\right\}<\infty .
$$

Here and below we denote for $\mathbb{R}$-Banach spaces $\left(V,\|\cdot\|_{V}\right)$ and $\left(W,\|\cdot\|_{W}\right)$ by $\operatorname{Lip}(V, W)$ the set of all Lipschitz continuous functions from $V$ to $W$ and we denote for $\mathbb{R}$-Banach spaces $\left(V,\|\cdot\|_{V}\right)$ and $\left(W,\|\cdot\|_{W}\right)$ and a function $f \in \operatorname{Lip}(V, W)$ by $|f|_{\operatorname{Lip}(V, W)} \in[0, \infty)$ the Lipschitz semi-norm associated to $f$ (see (15) in Subsection 1.1 below for details). The finiteness of the second element in the set in the maximum in (9) follows from the perturbation estimate (5) (with $Y^{1}=X^{x}$ and $Y^{2}=X^{y}$ for $x, y \in H_{-\delta}, \delta \in[0, \hat{\delta})$ in the notation of (5) ) and the finiteness of the first element in the set in the maximum in (9) is a consequence from (7), which, in turn, also follows from the perturbation estimate (5) (see above and the proof of Corollary 2.10 for details). Roughly speaking, Corollary 2.10 establishes the existence of mild solutions of the SEE (8) and also establishes the Lipschitz continuity of the solutions with respect to the initial conditions for any initial condition in $H_{-\delta}$ and any $\delta<\hat{\delta}=\frac{1}{2}\left[1+\mathbb{1}_{\{0\}}\left(|B|_{\operatorname{Lip}\left(H, H S\left(U, H_{-\beta}\right)\right)}\right)\right]$ (see (9)). In Corollary 3.1, Proposition 3.2, Proposition 3.4, and Proposition 3.5 below we demonstrate that the regularity barrier

$$
\hat{\delta}=\frac{1}{2}\left[1+\mathbb{1}_{\{0\}}\left(|B|_{\operatorname{Lip}\left(H, H S\left(U, H_{-\beta}\right)\right)}\right)\right]= \begin{cases}1 / 2 & : B \text { is not a constant function } \\ 1 & : B \text { is a constant function }\end{cases}
$$

for the regularity of the initial conditions revealed in Corollary 2.10 (and Proposition 2.7 and Theorem 2.9, respectively) can, in general, not essentially be improved. In particular, Corollary 3.1 and Proposition 3.2 below prove in the case where $H=U=L^{2}((0,1) ; \mathbb{R})$, where $\beta \in(1 / 4,1 / 2)$, where $A: D(A) \subseteq H \rightarrow H$ is the Laplacian with periodic boundary conditions on $H$, and where $B \in L\left(H, H S\left(H, H_{-\beta}\right)\right)$ satisfies $\forall u, v \in H: B(v) u=v \cdot u$ ( $B$ is not a constant function) that it holds (i) that there exist up-to-modifications unique $\left(\mathcal{F}_{t}\right)_{t \in[0, T]}$-predictable 
stochastic processes $X^{x}:[0, T] \times \Omega \rightarrow H_{-\delta}, x \in H_{-\delta}, \delta \in[0,1 / 2)$, which fulfill for all $q \in[2, \infty)$, $\delta \in[0,1 / 2), x \in H_{-\delta}, t \in[0, T]$ that $X^{x}((0, T] \times \Omega) \subseteq H$, that $\sup _{s \in(0, T]} s^{\delta}\left\|X_{s}^{x}\right\|_{L^{q}(\mathbb{P} ; H)}<\infty$, and $\mathbb{P}$-a.s. that

$$
X_{t}^{x}=e^{t A} x+\int_{0}^{t} e^{(t-s) A} B\left(X_{s}^{x}\right) \mathrm{d} W_{s}
$$

(ii) that

$$
\forall \delta \in[0,1 / 2), q \in[2, \infty), t \in(0, T]: \sup _{\substack{x, y \in H, x \neq y}}\left[\frac{\left\|X_{t}^{x}-X_{t}^{y}\right\|_{L^{q}(\mathbb{P} ; H)}}{\|x-y\|_{H_{-\delta}}}\right]<\infty,
$$

and (iii) that

$$
\forall \delta \in(1 / 2, \infty), q \in[2, \infty), t \in(0, T]: \sup _{\substack{x, y \in H, x \neq y}}\left[\frac{\left\|X_{t}^{x}-X_{t}^{y}\right\|_{L^{q}(\mathbb{P} ; H)}}{\|x-y\|_{H_{-\delta}}}\right]=\infty .
$$

The SEE (11) is sometimes referred to as a continuous version of the parabolic Anderson model in the literature (see, e.g., Carmona \& Molchanov [6]). In addition, Proposition 3.2 below disproves the existence of square integrable solutions of the SEE (11) with initial conditions in $\left(\cup_{\delta \in \mathbb{R}} H_{\delta}\right) \backslash H_{-1 / 2}$. The noise in the counterexample SEE (11) is spatially very rough and one might question whether the regularity barrier (10) can be overcome in the case of more regular spatially smooth noise. In Proposition 3.4 below we answer this question to the negative by presenting another counterexample SEE with a non-constant diffusion coefficient but a spatially smooth noise for which we disprove the existence of square integrable solutions with initial conditions in $\left(\cup_{\delta \in \mathbb{R}} H_{\delta}\right) \backslash H_{-1 / 2}$ (cf., however, also Proposition 3.3 below). Proposition 3.5 below also provides a further counterexample SEE which illustrates the sharpness of the regularity barrier (10) in the case where $B$ is a constant function.

Proposition 2.7, Theorem 2.9, and Corollary 2.10 outlined above (see (5)-(9)) are of particular importance for establishing regularity properties for Kolmogorov backward equations associated to parabolic semilinear SEEs and, thereby, for establishing essentially sharp probabilistically weak convergence rates for numerical approximations of parabolic semilinear SEEs (cf., e.g., Lemmas 4.4-4.6 in Debussche [12], Lemma 3.3 in Wang \& Gan [30], (4.2)-(4.3) in Andersson \& Larsson [1], Propositions 5.1-5.2 and Lemma 5.4 in Bréhier [2], Lemma 3.3 in Wang [29], (79) in Conus et al. 9], Proposition 7.1, Lemma 10.5, and Lemma 10.10 in Kopec [20], and (183)-(184) in Jentzen \& Kurniawan [17]). The analytically weak norm for the initial condition in (9) as well as the singularities in the nonlinear coefficients of the SEE in (11) and (2) above translate in an analytically weak norm for the approximation errors in the probabilistically weak error analysis which, in turn, results in essentially sharp probabilistically weak convergence rates (cf., e.g., Theorem 2.2 in Debussche [12], Theorem 2.1 in Wang \& Gan [30], Theorem 1.1 in Andersson \& Larsson [1], Theorem 1.1 in Bréhier [2], Theorem 5.1 in Bréhier \& Kopec [3], Corollary 1 in Wang [29], Corollary 5.2 in Conus et al. [9], Theorem 6.1 in Kopec [20], and Corollary 8.2 in [17]). The perturbation inequality in Proposition 2.7 (see (5) above) is also useful to establish essentially sharp probabilistically strong convergence rates for numerical approximations and perturbations of SEEs (cf., e.g., Proposition 4.1 in Conus et al. 9] and Proposition 4.3 in [17]). 


\subsection{Notation}

Throughout this article the following notation is used. For two measurable spaces $(A, \mathcal{A})$ and $(B, \mathcal{B})$ we denote by $\mathcal{M}(\mathcal{A}, \mathcal{B})$ the set of all $\mathcal{A} / \mathcal{B}$-measurable functions. For a set $A$ we denote by $\mathcal{P}(A)$ the power set of $A$ and we denote by $\#_{A}: \mathcal{P}(A) \rightarrow[0, \infty]$ the counting measure on $A$. For a Borel measurable set $A \in \mathcal{B}(\mathbb{R})$ we denote by $\mu_{A}: \mathcal{B}(A) \rightarrow[0, \infty]$ the Lebesgue-Borel measure on $A$. For a real number $T \in(0, \infty)$ and a probability space $(\Omega, \mathcal{F}, \mathbb{P})$ with a normal filtration $\left(\mathcal{F}_{t}\right)_{t \in[0, T]}$ (see, e.g., Definition 2.1.11 in [23]) we call the quadruple $\left(\Omega, \mathcal{F}, \mathbb{P},\left(\mathcal{F}_{t}\right)_{t \in[0, T]}\right)$ a stochastic basis. For a real number $T \in(0, \infty)$ and a filtered probability space $\left(\Omega, \mathcal{F}, \mathbb{P},\left(\mathcal{F}_{t}\right)_{t \in[0, T]}\right)$ we denote by $\operatorname{Pred}\left(\left(\mathcal{F}_{t}\right)_{t \in[0, T]}\right)$ the sigma-algebra given by

$$
\operatorname{Pred}\left(\left(\mathcal{F}_{t}\right)_{t \in[0, T]}\right)=\sigma_{[0, T] \times \Omega}\left(\left\{(s, t] \times A: s, t \in[0, T], s<t, A \in \mathcal{F}_{s}\right\} \cup\left\{\{0\} \times A: A \in \mathcal{F}_{0}\right\}\right)
$$

(the predictable sigma-algebra associated to $\left.\left(\mathcal{F}_{t}\right)_{t \in[0, T]}\right)$. We denote by $\lceil\cdot\rceil_{h}: \mathbb{R} \rightarrow \mathbb{R}, h \in$ $(0, \infty)$, the functions which satisfy for all $h \in(0, \infty), t \in \mathbb{R}$ that $\lceil t\rceil_{h}=\min ([t, \infty) \cap\{0, h,-h$, $2 h,-2 h, \ldots\})$. For $\mathbb{R}$-Banach spaces $\left(V,\|\cdot\|_{V}\right)$ and $\left(W,\|\cdot\|_{W}\right)$ we denote by $|\cdot|_{\operatorname{Lip}(V, W)}: \mathcal{C}(V, W) \rightarrow$ $[0, \infty]$ and $\|\cdot\|_{\operatorname{Lip}(V, W)}: \mathcal{C}(V, W) \rightarrow[0, \infty]$ the functions which satisfy for all $f \in \mathcal{C}(V, W)$ that

$$
\begin{aligned}
|f|_{\operatorname{Lip}(V, W)} & =\sup \left(\left\{\frac{\|f(x)-f(y)\|_{W}}{\|x-y\|_{V}}: x, y \in V, x \neq y\right\} \cup\{0\}\right), \\
\|f\|_{\operatorname{Lip}(V, W)} & =\|f(0)\|_{W}+|f|_{\operatorname{Lip}(V, W)}
\end{aligned}
$$

and we denote by $\operatorname{Lip}(V, W)$ the set given by $\operatorname{Lip}(V, W)=\left\{f \in \mathcal{C}(V, W):|f|_{\operatorname{Lip}(V, W)}<\infty\right\}$. For a separable $\mathbb{R}$-Hilbert space $\left(H,\|\cdot\|_{H},\langle\cdot, \cdot\rangle_{H}\right)$, real numbers $T \in(0, \infty), \eta \in \mathbb{R}, r \in$ $[0, \infty), s \in[0,1]$, and a generator of a strongly continuous analytic semigroup $A: D(A) \subseteq$ $H \rightarrow H$ with $\operatorname{spectrum}(A) \subseteq\{z \in \mathbb{C}: \operatorname{Re}(z)<\eta\}$ we denote by $\chi_{A, \eta}^{r, T}, \kappa_{A, \eta}^{s, T} \in[0, \infty)$ the real numbers given by $\chi_{A, \eta}^{r, T}=\sup _{t \in(0, T]} t^{r}\left\|(\eta-A)^{r} e^{t A}\right\|_{L(H)}$ (cf., e.g., (3) in Section 1 above) and $\kappa_{A, \eta}^{s, T}=\sup _{t \in(0, T]} t^{-s}\left\|(\eta-A)^{-s}\left(e^{t A}-\operatorname{Id}_{H}\right)\right\|_{L(H)}$ (cf., e.g., [24, Lemma 11.36]) . We denote by $\mathbb{B}:(0, \infty)^{2} \rightarrow(0, \infty)$ the function with the property that for all $x, y \in(0, \infty)$ it holds that $\mathbb{B}(x, y)=\int_{0}^{1} t^{(x-1)}(1-t)^{(y-1)} \mathrm{d} t$ (Beta function). We denote by $\mathrm{E}_{\alpha, \beta}:[0, \infty) \rightarrow[0, \infty)$, $\alpha, \beta \in(-\infty, 1)$, the functions which satisfy for all $\alpha, \beta \in(-\infty, 1), x \in[0, \infty)$ that

$$
\mathrm{E}_{\alpha, \beta}[x]=1+\sum_{n=1}^{\infty} x^{n} \prod_{k=0}^{n-1} \mathbb{B}(1-\beta, k(1-\beta)+1-\alpha)
$$

(generalized exponential function; cf. Lemma 7.1.1 in Chapter 7 in Henry [14, (1.0.3) in Chapter 1 in Gorenflo et al. [13], and Lemma 2.6 below). For real numbers $T \in(0, \infty), \eta \in \mathbb{R}$, $p \in[1, \infty), a, \lambda \in(-\infty, 1), b \in\left(-\infty, \frac{1}{2}\right)$, a separable $\mathbb{R}$-Hilbert space $\left(H,\|\cdot\|_{H},\langle\cdot, \cdot\rangle_{H}\right)$, and a generator $A: D(A) \subseteq H \rightarrow H$ of a strongly continuous analytic semigroup with $\operatorname{spectrum}(A) \subseteq\{z \in \mathbb{C}: \operatorname{Re}(z)<\eta\}$ we denote by $\Theta_{A, \eta, p, T}^{a, b, \lambda}:[0, \infty)^{2} \rightarrow[0, \infty]$ the function 
which satisfies for all $L, \hat{L} \in[0, \infty)$ that

$$
\begin{aligned}
& \Theta_{A, \eta, p, T}^{a, b, \lambda}(L, \hat{L})= \\
& \left\{\begin{array}{ll}
\sqrt{2}\left|E_{2 \lambda, \max \{a, 2 b\}}\left[\left|\frac{\chi_{A, \eta}^{a, T} L \sqrt{2} T^{(1-a)}}{\sqrt{1-a}}+\chi_{A, \eta}^{b, T} \hat{L} \sqrt{p(p-1) T^{(1-2 b)}}\right|^{2}\right]\right|^{1 / 2} & :(\lambda, \hat{L}) \in\left(-\infty, \frac{1}{2}\right) \times(0, \infty) \\
E_{\lambda, a}\left[\chi_{A, \eta}^{a, T} L T^{(1-a)}\right] & : \hat{L}=0 \\
\infty & : \text { otherwise }
\end{array} .\right.
\end{aligned}
$$

For a measure space $(\Omega, \mathcal{F}, \mu)$, a measurable space $(S, \mathcal{S})$, and an $\mathcal{F} / \mathcal{S}$-measurable function $f: \Omega \rightarrow S$ we denote by $[f]_{\mu, \mathcal{S}}$ the set given by

$$
[f]_{\mu, \mathcal{S}}=\{g \in \mathcal{M}(\mathcal{F}, \mathcal{S}):(\exists A \in \mathcal{F}: \mu(A)=0 \text { and }\{\omega \in \Omega: f(\omega) \neq g(\omega)\} \subseteq A)\} .
$$

For a measure space $(\Omega, \mathcal{F}, \mu)$ and a measurable space $(S, \mathcal{S})$ we do as usual often not distinguish between an $\mathcal{F} / \mathcal{S}$-measurable function $f: \Omega \rightarrow S$ and its equivalence class $[f]_{\mu, \mathcal{S}}$.

\section{Stochastic evolution equations (SEEs) with singulari- ties at the initial time}

\subsection{Setting}

Throughout this section the following setting is frequently used. Let $\left(H,\|\cdot\|_{H},\langle\cdot, \cdot\rangle_{H}\right)$ and $\left(U,\|\cdot\|_{U},\langle\cdot, \cdot\rangle_{U}\right)$ be separable $\mathbb{R}$-Hilbert spaces with $\#_{H}(H)>1$, let $T \in(0, \infty), \eta \in \mathbb{R}$, $p \in[2, \infty), \alpha \in[0,1), \hat{\alpha} \in(-\infty, 1), \beta \in[0,1 / 2), \hat{\beta} \in(-\infty, 1 / 2), L_{0}, \hat{L}_{0}, L_{1}, \hat{L}_{1} \in[0, \infty)$ satisfy $\mathbb{1}_{(0, \infty)}\left(L_{1}\right) \cdot[\alpha+\hat{\alpha}]<3 / 2$, let $\left(\Omega, \mathcal{F}, \mathbb{P},\left(\mathcal{F}_{t}\right)_{t \in[0, T]}\right)$ be a stochastic basis, let $\left(W_{t}\right)_{t \in[0, T]}$ be an $\operatorname{Id}_{U}$-cylindrical $\left(\mathcal{F}_{t}\right)_{t \in[0, T]}$-Wiener process, let $A: D(A) \subseteq H \rightarrow H$ be a generator of a strongly continuous analytic semigroup with $\operatorname{spectrum}(A) \subseteq\{z \in \mathbb{C}: \operatorname{Re}(z)<\eta\}$, let $\left(H_{r},\|\cdot\|_{H_{r}},\langle\cdot, \cdot\rangle_{H_{r}}\right), r \in \mathbb{R}$, be a family of interpolation spaces associated to $\eta-A$, and let $\mathbf{F} \in$ $\mathcal{M}\left(\operatorname{Pred}\left(\left(\mathcal{F}_{t}\right)_{t \in[0, T]}\right) \otimes \mathcal{B}(H), \mathcal{B}\left(H_{-\alpha}\right)\right)$ and $\mathbf{B} \in \mathcal{M}\left(\operatorname{Pred}\left(\left(\mathcal{F}_{t}\right)_{t \in[0, T]}\right) \otimes \mathcal{B}(H), \mathcal{B}\left(H S\left(U, H_{-\beta}\right)\right)\right)$ satisfy for all $t \in(0, T], X, Y \in \mathcal{L}^{p}(\mathbb{P} ; H)$ that

$$
\begin{gathered}
\|\mathbf{F}(t, X)-\mathbf{F}(t, Y)\|_{L^{p}\left(\mathbb{P} ; H_{-\alpha}\right)} \leq L_{0}\|X-Y\|_{L^{p}(\mathbb{P} ; H)},\|\mathbf{F}(t, 0)\|_{L^{p}\left(\mathbb{P} ; H_{-\alpha}\right)} \leq \hat{L}_{0} t^{-\hat{\alpha}} \\
\|\mathbf{B}(t, X)-\mathbf{B}(t, Y)\|_{L^{p}\left(\mathbb{P} ; H S\left(U, H_{-\beta}\right)\right)} \leq L_{1}\|X-Y\|_{L^{p}(\mathbb{P} ; H)},\|\mathbf{B}(t, 0)\|_{L^{p}\left(\mathbb{P} ; H S\left(U, H_{-\beta}\right)\right)} \leq \hat{L}_{1} t^{-\hat{\beta}}
\end{gathered}
$$

\subsection{Predictable stochastic processes with singularities at the initial time}

The next result, Lemma 2.1, is an elementary lemma that slightly generalizes Proposition 3.6 (ii) in Da Prato \& Zabczyk [10]. 
Lemma 2.1 (Existence of predictable modifications). Let $T \in[0, \infty)$, let $\left(\Omega, \mathcal{F}, \mathbb{P},\left(\mathcal{F}_{t}\right)_{t \in[0, T]}\right)$ be a stochastic basis, let $\left(E, d_{E}\right)$ be a complete and separable metric space, and let $Y:[0, T] \times \Omega \rightarrow E$ be an $\left(\mathcal{F}_{t}\right)_{t \in[0, T]}$-adapted stochastic process which satisfies for all $t \in(0, \infty) \cap(-\infty, T]$ that $\lim \sup _{[0, T] \ni s \rightarrow t} \mathbb{E}\left[\min \left\{1, d_{E}\left(Y_{s}, Y_{t}\right)\right\}\right]=0$. Then there exists an $\left(\mathcal{F}_{t}\right)_{t \in[0, T]}$-predictable stochastic process $X:[0, T] \times \Omega \rightarrow E$ which satisfies for all $t \in[0, T]$ that $\mathbb{P}\left(X_{t}=Y_{t}\right)=1$.

Proof. First, we observe that the assumption that $(\Omega, \mathcal{F}, \mathbb{P})$ is a probability space ensures that $\Omega \neq \emptyset$ and this implies that $[0, T] \times \Omega \neq \emptyset$. The assumption that $Y:[0, T] \times \Omega \rightarrow E$ is a mapping from $[0, T] \times \Omega$ to $E$ therefore ensures that $E \neq \emptyset$. Hence, there exists an element $e_{0} \in E$. In the next step assume without loss of generality that $T>0$, let $Z^{N}:[0, T] \times \Omega \rightarrow E, N \in \mathbb{N}$, be the functions with the property that for all $N \in \mathbb{N}, t \in[0, T]$ it holds that $Z_{t}^{N}=Y_{\max \left\{[t]_{T / N}-T / N, 0\right\}}$, and let $w:(0, T] \times \mathbb{N} \rightarrow[0, \infty)$ be the function with the property that for all $\varepsilon \in(0, T], N \in \mathbb{N}$ it holds that

$$
w(\varepsilon, N)=\sup _{\substack{t_{1}, t_{2} \in[\varepsilon, T],\left|t_{1}-t_{2}\right| \leq T / N}} \mathbb{E}\left[\min \left\{1, d_{E}\left(Y_{t_{1}}, Y_{t_{2}}\right)\right\}\right] .
$$

The assumption that $\forall t \in(0, T]: \lim _{s \rightarrow t} \mathbb{E}\left[\min \left\{1, d_{E}\left(Y_{s}, Y_{t}\right)\right\}\right]=0$ ensures that for all $\varepsilon \in(0, T]$ it holds that $\lim _{N \rightarrow \infty} w(\varepsilon, N)=0$. This implies that there exists a strictly increasing sequence $N_{k} \in \mathbb{N}, k \in \mathbb{N}$, with the property that for all $k \in \mathbb{N}$ it holds that

$$
w\left(\frac{1}{k}, N_{k}\right)<\frac{1}{2^{k}}
$$

Next let $X:[0, T] \times \Omega \rightarrow E$ be the mapping with the property that for all $(t, \omega) \in[0, T] \times \Omega$ it holds that

$$
X_{t}(\omega)= \begin{cases}\lim _{k \rightarrow \infty} Z_{t}^{N_{k}}(\omega) & :\left(Z_{t}^{N_{k}}(\omega)\right)_{k \in \mathbb{N}} \text { is convergent } \\ e_{0} & : \text { else }\end{cases}
$$

The fact that for all $N \in \mathbb{N}$ it holds that $Z^{N}$ is $\operatorname{Pred}\left(\left(\mathcal{F}_{t}\right)_{t \in[0, T]}\right) / \mathcal{B}(E)$-measurable, the assumption that $\left(E, d_{E}\right)$ is complete and separable, and, e.g., Exercise 1.74 in Chapter 1 in Hoffmann-Jørgensen [15] imply that

$$
\left\{(t, \omega) \in[0, T] \times \Omega:\left(Z_{t}^{N_{k}}(\omega)\right)_{k \in \mathbb{N}} \text { is convergent }\right\} \in \operatorname{Pred}\left(\left(\mathcal{F}_{t}\right)_{t \in[0, T]}\right) .
$$

This together with the fact that for all $N \in \mathbb{N}$ it holds that $Z^{N}$ is $\operatorname{Pred}\left(\left(\mathcal{F}_{t}\right)_{t \in[0, T]}\right) / \mathcal{B}(E)$ measurable, and, e.g., Exercise 1.74 in Chapter 1 in Hoffmann-Jørgensen [15] ensure that $X$ is $\operatorname{Pred}\left(\left(\mathcal{F}_{t}\right)_{t \in[0, T]}\right) / \mathcal{B}(E)$-measurable. It thus remains to prove that $X$ is a modification of $Y$. For this we note that for all $N \in \mathbb{N}, t \in\left(\frac{T}{N}, T\right]$ it holds that

$$
\mathbb{E}\left[\min \left\{1, d_{E}\left(Y_{t}, Z_{t}^{N}\right)\right\}\right]=\mathbb{E}\left[\min \left\{1, d_{E}\left(Y_{t}, Y_{\lceil t\rceil_{T / N}-T / N}\right)\right\}\right] \leq w\left(t-\frac{T}{N}, N\right) .
$$

This together with (22), the fact that $\forall \varepsilon_{1}, \varepsilon_{2} \in(0, T], N \in \mathbb{N}$ with $\varepsilon_{1} \leq \varepsilon_{2}: w\left(\varepsilon_{1}, N\right) \geq$ $w\left(\varepsilon_{2}, N\right)$, and the fact that $\forall t \in(0, T], k \in \mathbb{N} \cap\left(\frac{T+1}{t}, \infty\right): \frac{1}{k}<t-\frac{T}{N_{k}}$ ensure that for all 
$t \in(0, T]$ it holds that

$$
\begin{aligned}
& \sum_{k=1}^{\infty} \mathbb{E}\left[\min \left\{1, d_{E}\left(Y_{t}, Z_{t}^{N_{k}}\right)\right\}\right]=\sum_{k \in \mathbb{N}} \mathbb{E}\left[\min \left\{1, d_{E}\left(Y_{t}, Y_{\lceil t\rceil_{T / N_{k}}-T / N_{k}}\right)\right\}\right] \\
& =\sum_{k \in \mathbb{N} \cap(0,(T+1) / t]} \mathbb{E}\left[\min \left\{1, d_{E}\left(Y_{t}, Y_{\lceil t\rceil_{T / N_{k}}-T / N_{k}}\right)\right\}\right] \\
& \quad+\sum_{k \in \mathbb{N} \cap((T+1) / t, \infty)} \mathbb{E}\left[\min \left\{1, d_{E}\left(Y_{t}, Y_{\lceil t\rceil_{T / N_{k}}-T / N_{k}}\right)\right\}\right] \\
& \leq \frac{T+1}{t}+\sum_{k \in \mathbb{N} \cap((T+1) / t, \infty)} w\left(t-\frac{T}{N_{k}}, N_{k}\right) \leq \frac{T+1}{t}+\sum_{k \in \mathbb{N} \cap((T+1) / t, \infty)} w\left(\frac{1}{k}, N_{k}\right) \\
& \leq \frac{T+1}{t}+\sum_{k \in \mathbb{N} \cap((T+1) / t, \infty)} \frac{1}{2^{k}}<\infty .
\end{aligned}
$$

This implies that for all $t \in(0, T]$ it holds $\mathbb{P}$-a.s. that $\lim \sup _{k \rightarrow \infty} d_{E}\left(Z_{t}^{N_{k}}, Y_{t}\right)=0$ (see, e.g., item (ii) of Theorem 6.12 in Klenke [19]). This and (23) ensure for all $t \in(0, T]$ that $\mathbb{P}\left(X_{t}=\right.$ $\left.Y_{t}\right)=1$. This and the fact that $\forall N \in \mathbb{N}: X_{0}=Z_{0}^{N}=Y_{0}$ complete the proof of Lemma 2.1.

Lemma 2.2. Let $\left(V_{k},\|\cdot\|_{V_{k}}\right), k \in\{0,1\}$, be separable $\mathbb{R}$-Banach spaces with $V_{1} \subseteq V_{0}$ continuously. Then

$$
\mathcal{B}\left(V_{1}\right)=\left\{B \in \mathcal{P}\left(V_{1}\right):\left(\exists A \in \mathcal{B}\left(V_{0}\right): B=A \cap V_{1}\right)\right\} \subseteq \mathcal{B}\left(V_{0}\right) .
$$

Proof. Throughout this proof let $\varphi: V_{1} \rightarrow V_{0}$ and $\phi: V_{1} \rightarrow V_{1}$ be the mappings with the property that for all $v \in V_{1}$ it holds that $\varphi(v)=\phi(v)=v$. Next observe that $\varphi \in \mathcal{C}\left(V_{1}, V_{0}\right)$. This implies that $\varphi \in \mathcal{M}\left(\mathcal{B}\left(V_{1}\right), \mathcal{B}\left(V_{0}\right)\right)$. Hence, we obtain that

$$
\left\{B \in \mathcal{P}\left(V_{1}\right):\left(\exists A \in \mathcal{B}\left(V_{0}\right): B=A \cap V_{1}\right)\right\} \subseteq \mathcal{B}\left(V_{1}\right) .
$$

Moreover, note that the fact that $\varphi \in \mathcal{M}\left(\mathcal{B}\left(V_{1}\right), \mathcal{B}\left(V_{0}\right)\right)$ allows us to apply, e.g., Theorem 2.4 in Chapter V in Parthasarathy [22] (with $(X, \mathscr{B})=\left(V_{1}, \mathcal{B}\left(V_{1}\right)\right),(Y, \mathscr{C})=\left(V_{0}, \mathcal{B}\left(V_{0}\right)\right.$ ), and $\varphi=\varphi$ in the notation of Theorem 2.4 in Chapter $V$ in Parthasarathy [22]) to obtain that for all $C \in \mathcal{B}\left(V_{1}\right)$ it holds that $V_{1}=\varphi\left(V_{1}\right) \in \mathcal{B}\left(V_{0}\right)$ and $C=\phi(C)=\left(\phi^{-1}\right)^{-1}(C) \in\{B \in$ $\left.\mathcal{P}\left(V_{1}\right):\left(\exists A \in \mathcal{B}\left(V_{0}\right): B=A \cap V_{1}\right)\right\}$. This implies that

$$
\mathcal{B}\left(V_{1}\right) \subseteq\left\{B \in \mathcal{P}\left(V_{1}\right):\left(\exists A \in \mathcal{B}\left(V_{0}\right): B=A \cap V_{1}\right)\right\} .
$$

Combining (28), (29), and the fact that $V_{1} \in \mathcal{B}\left(V_{0}\right)$ completes the proof of Lemma 2.2.

Lemma 2.3 (Non-stochastic integral). Assume the setting in Section $[2.1$, let $\delta \in \mathbb{R}, \lambda \in$ $(-\infty, 1)$, and let $Y:[0, T] \times \Omega \rightarrow H_{\delta}$ be an $\left(\mathcal{F}_{t}\right)_{t \in[0, T]}$-predictable stochastic process which satisfies $Y((0, T] \times \Omega) \subseteq H$ and $\sup _{t \in(0, T]} t^{\lambda}\left\|Y_{t}\right\|_{L^{p}(\mathbb{P} ; H)}<\infty$. Then

(i) for all $t \in[0, T]$ it holds $\mathbb{P}$-a.s. that $\int_{0}^{t}\left\|e^{(t-s) A} \mathbf{F}\left(s, Y_{s}\right)\right\|_{H} d s<\infty$, 
(ii) there exists an up-to-modifications unique $\left(\mathcal{F}_{t}\right)_{t \in[0, T]}$-predictable stochastic process $\bar{Y}:[0, T] \times$ $\Omega \rightarrow H$ such that for all $t \in[0, T]$ it holds $\mathbb{P}$-a.s. that $\bar{Y}_{t}=\int_{0}^{t} e^{(t-s) A} \mathbf{F}\left(s, Y_{s}\right) d s$,

(iii) it holds that

$$
\begin{aligned}
& \sup _{t \in(0, T]} t^{(\max \{\lambda, \hat{\alpha}\}+\alpha-1)}\left\|\bar{Y}_{t}\right\|_{L^{p}(\mathbb{P} ; H)} \leq\left(\hat{L}_{0}+L_{0} \sup _{t \in(0, T]} t^{\lambda}\left\|Y_{t}\right\|_{L^{p}(\mathbb{P} ; H)}\right) \\
& \cdot|T \vee 1|^{|\lambda-\hat{\alpha}|} \mathbb{B}(1-\alpha, 1-\max \{\lambda, \hat{\alpha}\}) \chi_{A, \eta}^{\alpha, T}<\infty,
\end{aligned}
$$

(iv) and for all $\varrho \in[0,1-\alpha), s, t \in(0, T]$ with $s<t$ it holds that

$$
\begin{aligned}
& \left\|\bar{Y}_{t}-\bar{Y}_{s}\right\|_{L^{p}(\mathbb{P} ; H)} \leq|T \vee 1|^{|\lambda-\hat{\alpha}|}\left(\hat{L}_{0}+L_{0} \sup _{u \in(0, T]} u^{\lambda}\left\|Y_{u}\right\|_{L^{p}(\mathbb{P} ; H)}\right)|t-s|^{\varrho} \\
& \cdot\left[\frac{\chi_{A, \eta}^{\alpha, T}|t-s|^{(1-\alpha-\varrho)}}{(1-\alpha) \min \left\{s^{\max \{\lambda, \hat{\alpha}\}}, t^{\max \{\lambda, \hat{\alpha}\}}\right\}}+\frac{\kappa_{A, \eta}^{\varrho, T} \chi_{A, \eta}^{\varrho+\alpha, T} \mathbb{B}(1-\alpha-\varrho, 1-\max \{\lambda, \hat{\alpha}\})}{s^{(\varrho+\alpha+\max \{\lambda, \hat{\alpha}\}-1)}}\right] .
\end{aligned}
$$

Proof. Throughout this proof let $K \in[0, \infty)$ be the real number given by $K=\sup _{t \in(0, T]} t^{\lambda}\left\|Y_{t}\right\|_{L^{p}(\mathbb{P} ; H)}$. We observe that (19) implies that for all $t \in(0, T]$ it holds that

$$
\begin{aligned}
& \int_{0}^{t}\left\|e^{(t-s) A} \mathbf{F}\left(s, Y_{s}\right)\right\|_{L^{p}(\mathbb{P} ; H)} \mathrm{d} s \\
& \leq \chi_{A, \eta}^{\alpha, T} \int_{0}^{t}(t-s)^{-\alpha}\left(\left\|\mathbf{F}\left(s, Y_{s}\right)-\mathbf{F}(s, 0)\right\|_{L^{p}\left(\mathbb{P} ; H_{-\alpha}\right)}+\|\mathbf{F}(s, 0)\|_{L^{p}\left(\mathbb{P} ; H_{-\alpha}\right)}\right) \mathrm{d} s \\
& \leq \chi_{A, \eta}^{\alpha, T} \int_{0}^{t}(t-s)^{-\alpha}\left(L_{0}\left\|Y_{s}\right\|_{L^{p}(\mathbb{P} ; H)}+\hat{L}_{0} s^{-\hat{\alpha}}\right) \mathrm{d} s \\
& \leq\left(K L_{0}+\hat{L}_{0}\right) \chi_{A, \eta}^{\alpha, T} \int_{0}^{t}(t-s)^{-\alpha} \max \left\{s^{-\lambda}, s^{-\hat{\alpha}}\right\} \mathrm{d} s \\
& \leq\left(K L_{0}+\hat{L}_{0}\right) \chi_{A, \eta}^{\alpha, T}|T \vee 1|^{|\lambda-\hat{\alpha}|} \int_{0}^{t}(t-s)^{-\alpha} s^{-\max \{\lambda, \hat{\alpha}\}} \mathrm{d} s \\
& \leq\left(K L_{0}+\hat{L}_{0}\right) \chi_{A, \eta}^{\alpha, T}|T \vee 1|^{|\lambda-\hat{\alpha}|} \mathbb{B}(1-\alpha, 1-\max \{\lambda, \hat{\alpha}\}) t^{(1-\alpha-\max \{\lambda, \hat{\alpha}\})} .
\end{aligned}
$$

In particular, this ensures that for all $t \in[0, T]$ it holds $\mathbb{P}$-a.s. that $\int_{0}^{t}\left\|e^{(t-s) A} \mathbf{F}\left(s, Y_{s}\right)\right\|_{H} d s<\infty$. Moreover, we note that for all $\varrho \in[0,1-\alpha), t_{1}, t_{2} \in(0, T]$ with $t_{1}<t_{2}$ it holds that

$$
\begin{aligned}
& \left\|\int_{0}^{t_{2}} e^{\left(t_{2}-s\right) A} \mathbf{F}\left(s, Y_{s}\right) \mathrm{d} s-\int_{0}^{t_{1}} e^{\left(t_{1}-s\right) A} \mathbf{F}\left(s, Y_{s}\right) \mathrm{d} s\right\|_{L^{p}(\mathbb{P} ; H)} \\
& \leq \int_{0}^{t_{1}}\left\|\left(e^{\left(t_{2}-s\right) A}-e^{\left(t_{1}-s\right) A}\right) \mathbf{F}\left(s, Y_{s}\right)\right\|_{L^{p}(\mathbb{P} ; H)} \mathrm{d} s+\int_{t_{1}}^{t_{2}}\left\|e^{\left(t_{2}-s\right) A} \mathbf{F}\left(s, Y_{s}\right)\right\|_{L^{p}(\mathbb{P} ; H)} \mathrm{d} s \\
& \leq\left\|\left(\operatorname{Id}_{H}-e^{\left(t_{2}-t_{1}\right) A}\right)\right\|_{L_{\left(H_{\varrho}, H\right)}} \int_{0}^{t_{1}}\left\|e^{\left(t_{1}-s\right) A}\right\|_{L_{\left(H_{-\alpha}, H_{\varrho}\right)}\left\|\mathbf{F}\left(s, Y_{s}\right)\right\|_{L^{p}\left(\mathbb{P} ; H_{-\alpha}\right)} \mathrm{d} s} \\
& \quad+\int_{t_{1}}^{t_{2}}\left\|e^{\left(t_{2}-s\right) A}\right\|_{L_{\left(H_{-\alpha}, H\right)}}\left\|\mathbf{F}\left(s, Y_{s}\right)\right\|_{L^{p}\left(\mathbb{P} ; H_{-\alpha}\right)} \mathrm{d} s .
\end{aligned}
$$


Assumption (19) hence implies that for all $\varrho \in[0,1-\alpha), t_{1}, t_{2} \in(0, T]$ with $t_{1}<t_{2}$ it holds that

$$
\begin{aligned}
& \left\|\int_{0}^{t_{2}} e^{\left(t_{2}-s\right) A} \mathbf{F}\left(s, Y_{s}\right) \mathrm{d} s-\int_{0}^{t_{1}} e^{\left(t_{1}-s\right) A} \mathbf{F}\left(s, Y_{s}\right) \mathrm{d} s\right\|_{L^{p}(\mathbb{P} ; H)} \\
& \leq \kappa_{A, \eta}^{\varrho, T} \chi_{A, \eta}^{\varrho+\alpha, T}\left|t_{2}-t_{1}\right|^{\varrho} \int_{0}^{t_{1}}\left(t_{1}-s\right)^{-(\alpha+\varrho)}\left(L_{0}\left\|Y_{s}\right\|_{L^{p}(\mathbb{P} ; H)}+\hat{L}_{0} s^{-\hat{\alpha}}\right) \mathrm{d} s \\
& +\chi_{A, \eta}^{\alpha, T} \int_{t_{1}}^{t_{2}}\left(t_{2}-s\right)^{-\alpha}\left(L_{0}\left\|Y_{s}\right\|_{L^{p}(\mathbb{P} ; H)}+\hat{L}_{0} s^{-\hat{\alpha}}\right) \mathrm{d} s \\
& \leq\left(K L_{0}+\hat{L}_{0}\right) \kappa_{A, \eta}^{\varrho, T} \chi_{A, \eta}^{\varrho+\alpha, T}\left|t_{2}-t_{1}\right|^{\varrho} \int_{0}^{t_{1}}\left(t_{1}-s\right)^{-(\alpha+\varrho)} \max \left\{s^{-\lambda}, s^{-\hat{\alpha}}\right\} \mathrm{d} s \\
& +\left(K L_{0}+\hat{L}_{0}\right) \chi_{A, \eta}^{\alpha, T} \int_{t_{1}}^{t_{2}}\left(t_{2}-s\right)^{-\alpha} \max \left\{s^{-\lambda}, s^{-\hat{\alpha}}\right\} \mathrm{d} s \\
& \leq\left(K L_{0}+\hat{L}_{0}\right)|T \vee 1|^{|\lambda-\hat{\alpha}|}\left[\chi_{A, \eta}^{\alpha, T} \int_{t_{1}}^{t_{2}}\left(t_{2}-s\right)^{-\alpha} s^{-\max \{\lambda, \hat{\alpha}\}} \mathrm{d} s\right. \\
& \left.+\kappa_{A, \eta}^{\varrho, T} \chi_{A, \eta}^{\varrho+\alpha, T}\left|t_{2}-t_{1}\right|^{\varrho} \int_{0}^{t_{1}}\left(t_{1}-s\right)^{-(\alpha+\varrho)} s^{-\max \{\lambda, \hat{\alpha}\}} \mathrm{d} s\right] \\
& \leq\left[\frac{\chi_{A, \eta}^{\alpha, T}\left|t_{2}-t_{1}\right|^{(1-\alpha)}}{(1-\alpha) \min \left\{\left|t_{1}\right|^{\left.\max \{\lambda, \hat{\alpha}\},\left|t_{2}\right|^{\max \{\lambda, \hat{\alpha}\}}\right\}}\right.}+\frac{\kappa_{A, \eta}^{\varrho, T} \chi_{A, \eta}^{\varrho+\alpha, T}\left|t_{2}-t_{1}\right|^{\varrho} \mathbb{B}(1-\alpha-\varrho, 1-\max \{\lambda, \hat{\alpha}\})}{\left|t_{1}\right|^{(\varrho+\alpha+\max \{\lambda, \hat{\alpha}\}-1)}}\right] \\
& \text { - }\left(K L_{0}+\hat{L}_{0}\right)|T \vee 1|^{|\lambda-\hat{\alpha}|} \text {. }
\end{aligned}
$$

Combining (32), (34), and Lemma 2.1 completes the proof of Lemma 2.3.

Lemma 2.4 (Stochastic integral). Assume the setting in Section 2.1, let $\delta, \lambda \in \mathbb{R}, \rho=\max \{\lambda+$ $\left.(\hat{\beta}-\lambda) \mathbb{1}_{\{0\}}\left(L_{1}\right), \hat{\beta}\right\}$ satisfy $\lambda \mathbb{1}_{(0, \infty)}\left(L_{1}\right)<1 / 2$, and let $Y:[0, T] \times \Omega \rightarrow H_{\delta}$ be an $\left(\mathcal{F}_{t}\right)_{t \in[0, T]^{-}}$ predictable stochastic process which satisfies $Y((0, T] \times \Omega) \subseteq H$ and $\sup _{t \in(0, T]} t^{\lambda}\left\|Y_{t}\right\|_{L^{p}(\mathbb{P} ; H)}<\infty$. Then

(i) for all $t \in[0, T]$ it holds $\mathbb{P}$-a.s. that $\int_{0}^{t}\left\|e^{(t-s) A} \mathbf{B}\left(s, Y_{s}\right)\right\|_{H S(U, H)}^{2} d s<\infty$,

(ii) there exists an up-to-modifications unique $\left(\mathcal{F}_{t}\right)_{t \in[0, T]}$-predictable stochastic process $\bar{Y}:[0, T] \times$ $\Omega \rightarrow H$ such that for all $t \in[0, T]$ it holds $\mathbb{P}$-a.s. that $\bar{Y}_{t}=\int_{0}^{t} e^{(t-s) A} \mathbf{B}\left(s, Y_{s}\right) \mathrm{d} W_{s}$,

(iii) it holds that

$$
\begin{aligned}
& \sup _{t \in(0, T]} t^{(\rho+\beta-1 / 2)}\left\|\bar{Y}_{t}\right\|_{L^{p}(\mathbb{P} ; H)} \leq \sqrt{\frac{p(p-1)}{2} \mathbb{B}(1-2 \beta, 1-2 \rho)} \\
& \cdot|T \vee 1|^{|\lambda-\hat{\beta}| \mathbb{1}_{(0, \infty)}\left(L_{1}\right)} \chi_{A, \eta}^{\beta, T}\left(\hat{L}_{1}+L_{1} \sup _{t \in(0, T]} t^{\lambda}\left\|Y_{t}\right\|_{L^{p}(\mathbb{P} ; H)}\right)<\infty,
\end{aligned}
$$

(iv) and for all $\varrho \in[0,1 / 2-\beta), s, t \in(0, T]$ with $s<t$ it holds that

$$
\begin{aligned}
& \left\|\bar{Y}_{t}-\bar{Y}_{s}\right\|_{L^{p}(\mathbb{P} ; H)} \leq|T \vee 1|^{|\lambda-\hat{\beta}| \mathbb{1}_{(0, \infty)}\left(L_{1}\right)}\left(\hat{L}_{1}+L_{1} \sup _{u \in(0, T]} u^{\lambda}\left\|Y_{u}\right\|_{L^{p}(\mathbb{P} ; H)}\right)|t-s|^{\varrho} \\
& \cdot \sqrt{\frac{p(p-1)}{2}}\left[\frac{\chi_{A, \eta}^{\beta, T}|t-s|^{(1 / 2-\beta-\varrho)}}{\min \left\{s^{\rho}, t^{\rho}\right\} \sqrt{1-2 \beta}}+\frac{\kappa_{A, \eta}^{\varrho, T} \chi_{A, \eta}^{\varrho+\beta, T}|\mathbb{B}(1-2 \beta-2 \varrho, 1-2 \rho)|^{1 / 2}}{s^{(\rho+\varrho+\beta-1 / 2)}}\right] .
\end{aligned}
$$


Proof. Throughout this proof let $K \in[0, \infty)$ be the real number given by $K=\sup _{t \in(0, T]} t^{\lambda}\left\|Y_{t}\right\|_{L^{p}(\mathbb{P} ; H)}$. We observe that (20) implies for all $t \in(0, T]$ that

$$
\begin{aligned}
& \int_{0}^{t}\left\|e^{(t-s) A} \mathbf{B}\left(s, Y_{s}\right)\right\|_{L^{p}(\mathbb{P} ; H S(U, H))}^{2} \mathrm{~d} s \\
& \leq\left|\chi_{A, \eta}^{\beta, T}\right|^{2} \int_{0}^{t}(t-s)^{-2 \beta}\left(L_{1}\left\|Y_{s}\right\|_{L^{p}(\mathbb{P} ; H)}+\hat{L}_{1} s^{-\hat{\beta}}\right)^{2} \mathrm{~d} s \\
& \leq\left|\chi_{A, \eta}^{\beta, T}\right|^{2}\left(K L_{1}+\hat{L}_{1}\right)^{2} \int_{0}^{t}(t-s)^{-2 \beta} \max \left\{s^{-2\left(\lambda+(\hat{\beta}-\lambda) \mathbb{1}_{\{0\}}\left(L_{1}\right)\right)}, s^{-2 \hat{\beta}}\right\} \mathrm{d} s \\
& \leq\left|\chi_{A, \eta}^{\beta, T}\right|^{2}\left(K L_{1}+\hat{L}_{1}\right)^{2}|T \vee 1|^{2|\lambda-\hat{\beta}| \mathbb{1}_{(0, \infty)}\left(L_{1}\right)} \int_{0}^{t}(t-s)^{-2 \beta} s^{-2 \rho} \mathrm{d} s \\
& \leq\left|\chi_{A, \eta}^{\beta, T}\right|^{2}\left(K L_{1}+\hat{L}_{1}\right)^{2}|T \vee 1|^{2|\lambda-\hat{\beta}| \mathbb{1}_{(0, \infty)}\left(L_{1}\right)} \mathbb{B}(1-2 \beta, 1-2 \rho) t^{(1-2 \beta-2 \rho)} .
\end{aligned}
$$

This implies, in particular, that for all $t \in[0, T]$ it holds $\mathbb{P}$-a.s. that $\int_{0}^{t}\left\|e^{(t-s) A} \mathbf{B}\left(s, Y_{s}\right)\right\|_{H S(U, H)}^{2} d s$ $<\infty$. In addition, (37) and the Burkholder-Davis-Gundy type inequality in Lemma 7.7 in Da Prato \& Zabczyk [10] ensure that for all $t \in(0, T]$ it holds that

$$
\begin{aligned}
& \left\|\int_{0}^{t} e^{(t-s) A} \mathbf{B}\left(s, Y_{s}\right) d W_{s}\right\|_{L^{p}(\mathbb{P} ; H)} \\
& \leq\left[\frac{p(p-1)}{2} \int_{0}^{t}\left\|e^{(t-s) A} \mathbf{B}\left(s, Y_{s}\right)\right\|_{L^{p}(\mathbb{P} ; H S(U, H))}^{2} \mathrm{~d} s\right]^{1 / 2} \\
& \leq \chi_{A, \eta}^{\beta, T}\left(K L_{1}+\hat{L}_{1}\right)|T \vee 1|^{|\lambda-\hat{\beta}| \mathbb{1}_{(0, \infty)}\left(L_{1}\right)} \sqrt{\frac{p(p-1)}{2} \mathbb{B}(1-2 \beta, 1-2 \rho)} t^{(1 / 2-\beta-\rho)} .
\end{aligned}
$$

Furthermore, we observe that the Burkholder-Davis-Gundy type inequality in Lemma 7.7 in Da Prato \& Zabczyk [10] proves that for all $\varrho \in[0,1 / 2-\beta), t_{1}, t_{2} \in(0, T]$ with $t_{1}<t_{2}$ it holds that

$$
\begin{aligned}
& \left\|\int_{0}^{t_{2}} e^{\left(t_{2}-s\right) A} \mathbf{B}\left(s, Y_{s}\right) \mathrm{d} W_{s}-\int_{0}^{t_{1}} e^{\left(t_{1}-s\right) A} \mathbf{B}\left(s, Y_{s}\right) \mathrm{d} W_{s}\right\|_{L^{p}(\mathbb{P} ; H)} \\
& \leq\left[\frac{p(p-1)}{2} \int_{0}^{t_{1}}\left\|\left(\operatorname{Id}_{H}-e^{\left(t_{2}-t_{1}\right) A}\right) e^{\left(t_{1}-s\right) A} \mathbf{B}\left(s, Y_{s}\right)\right\|_{L^{p}(\mathbb{P} ; H S(U, H))}^{2} \mathrm{~d} s\right]^{1 / 2} \\
& +\left[\frac{p(p-1)}{2} \int_{t_{1}}^{t_{2}}\left\|e^{\left(t_{2}-s\right) A} \mathbf{B}\left(s, Y_{s}\right)\right\|_{L^{p}(\mathbb{P} ; H S(U, H))}^{2} \mathrm{~d} s\right]^{1 / 2} \\
& \leq\left[\frac{p(p-1)}{2} \int_{t_{1}}^{t_{2}}\left\|e^{\left(t_{2}-s\right) A}\right\|_{L\left(H_{-\beta}, H\right)}^{2}\left\|\mathbf{B}\left(s, Y_{s}\right)\right\|_{L^{p}\left(\mathbb{P} ; H S\left(U, H_{-\beta}\right)\right)}^{2} \mathrm{~d} s\right]^{1 / 2} \\
& \quad+\left[\frac{p(p-1)}{2} \int_{0}^{t_{1}}\left\|e^{\left(t_{1}-s\right) A}\right\|_{L_{\left(H_{-\beta}, H_{\varrho}\right)}^{2}}^{2}\left\|\mathbf{B}\left(s, Y_{s}\right)\right\|_{L^{p}\left(\mathbb{P} ; H S\left(U, H_{-\beta}\right)\right)}^{2} \mathrm{~d} s\right]^{1 / 2} \\
& \quad \cdot\left\|\left(\operatorname{Id}_{H}-e^{\left(t_{2}-t_{1}\right) A}\right)\right\|_{L\left(H_{\varrho}, H\right)} .
\end{aligned}
$$


Assumption (20) hence ensures that for all $\varrho \in[0,1 / 2-\beta), t_{1}, t_{2} \in(0, T]$ with $t_{1}<t_{2}$ it holds that

$$
\begin{aligned}
& \left\|\int_{0}^{t_{2}} e^{\left(t_{2}-s\right) A} \mathbf{B}\left(s, Y_{s}\right) \mathrm{d} W_{s}-\int_{0}^{t_{1}} e^{\left(t_{1}-s\right) A} \mathbf{B}\left(s, Y_{s}\right) \mathrm{d} W_{s}\right\|_{L^{p}(\mathbb{P} ; H)} \\
& \leq \kappa_{A, \eta}^{\varrho, T} \chi_{A, \eta}^{\varrho+\beta, T}\left|t_{2}-t_{1}\right|^{\varrho}\left[\frac{p(p-1)}{2} \int_{0}^{t_{1}}\left(t_{1}-s\right)^{-(2 \beta+2 \varrho)}\left(L_{1}\left\|Y_{s}\right\|_{L^{p}(\mathbb{P} ; H)}+\hat{L}_{1} s^{-\hat{\beta}}\right)^{2} \mathrm{~d} s\right]^{1 / 2} \\
& +\chi_{A, \eta}^{\beta, T}\left[\frac{p(p-1)}{2} \int_{t_{1}}^{t_{2}}\left(t_{2}-s\right)^{-2 \beta}\left(L_{1}\left\|Y_{s}\right\|_{L^{p}(\mathbb{P} ; H)}+\hat{L}_{1} s^{-\hat{\beta}}\right)^{2} \mathrm{~d} s\right]^{1 / 2} \\
& \leq \sqrt{\frac{p(p-1)}{2}} \kappa_{A, \eta}^{\varrho, T} \chi_{A, \eta}^{\varrho+\beta, T}\left(K L_{1}+\hat{L}_{1}\right)|T \vee 1|^{|\lambda-\hat{\beta}| \mathbb{1}_{(0, \infty)}\left(L_{1}\right)}\left|t_{2}-t_{1}\right|^{\varrho} \\
& \cdot\left[\int_{0}^{t_{1}}\left(t_{1}-s\right)^{-(2 \beta+2 \varrho)} s^{-2 \rho} \mathrm{d} s\right]^{1 / 2}+\frac{\chi_{A, \eta}^{\beta, T}|T \vee 1|^{|\lambda-\hat{\beta}| \mathbb{1}(0, \infty)}{ }^{\left(L_{1}\right)}\left(K L_{1}+\hat{L}_{1}\right) \sqrt{\frac{p(p-1)}{2}\left|t_{2}-t_{1}\right|^{(1-2 \beta)}}}{\min \left\{\left|t_{1}\right|^{\rho},\left|t_{2}\right|^{\rho}\right\} \sqrt{1-2 \beta}} \\
& \leq\left[\frac{\kappa_{A, \eta}^{\varrho, T} \chi_{A, \eta}^{\varrho+\beta, T}\left|t_{2}-t_{1}\right|^{\varrho} \sqrt{\mathbb{B}(1-2 \beta-2 \varrho, 1-2 \rho)}}{\left|t_{1}\right|^{(\rho+\varrho+\beta-1 / 2)}}+\frac{\chi_{A, T}^{\beta, T}\left|t_{2}-t_{1}\right|^{(1 / 2-\beta)}}{\min \left\{\left|t_{1}\right|^{\rho},\left|t_{2}\right|^{\rho}\right\} \sqrt{1-2 \beta}}\right] \\
& \cdot \sqrt{\frac{p(p-1)}{2}}|T \vee 1|^{|\lambda-\hat{\beta}| \mathbb{1}_{(0, \infty)}\left(L_{1}\right)}\left(K L_{1}+\hat{L}_{1}\right) \text {. }
\end{aligned}
$$

Combining (38), (40), and Lemma 2.1] completes the proof of Lemma 2.4 .

Lemma 2.5. Let $\left(V_{k},\|\cdot\|_{V_{k}}\right), k \in\{0,1\}$, be separable $\mathbb{R}$-Banach spaces with $V_{1} \subseteq V_{0}$ continuously and densely, let $T \in(0, \infty), \lambda \in \mathbb{R}, p \in[1, \infty)$, let $\left(\Omega, \mathcal{F}, \mathbb{P},\left(\mathcal{F}_{t}\right)_{t \in[0, T]}\right)$ be a filtered probability space, let $\mathcal{L} \subseteq \mathcal{M}\left(\operatorname{Pred}\left(\left(\mathcal{F}_{t}\right)_{t \in[0, T]}\right), \mathcal{B}\left(V_{0}\right)\right)$ be the set given by

$$
\mathcal{L}=\left\{\begin{array}{c}
X \in \mathcal{M}\left(\operatorname{Pred}\left(\left(\mathcal{F}_{t}\right)_{t \in[0, T]}\right), \mathcal{B}\left(V_{0}\right)\right): X((0, T] \times \Omega) \subseteq V_{1}, \\
\left\|X_{0}\right\|_{\mathcal{L}^{p}\left(\mathbb{P} ; V_{0}\right)}+\sup _{t \in(0, T]} t^{\lambda}\left\|X_{t}\right\|_{\mathcal{L}^{p}\left(\mathbb{P} ; V_{1}\right)}<\infty
\end{array}\right\}
$$

let $|\cdot|_{\mathcal{L}}: \mathcal{L} \rightarrow[0, \infty)$ be the mapping which satisfies for all $X \in \mathcal{L}$ that

$$
|X|_{\mathcal{L}}=\left\|X_{0}\right\|_{\mathcal{L}^{p}\left(\mathbb{P} ; V_{0}\right)}+\sup _{t \in(0, T]}\left[t^{\lambda}\left\|X_{t}\right\|_{\mathcal{L}^{p}\left(\mathbb{P} ; V_{1}\right)}\right]
$$

and let $X^{N} \in \mathcal{L}, N \in \mathbb{N}$, satisfy $\lim \sup _{N \rightarrow \infty} \sup _{n, m \in \mathbb{N} \cap[N, \infty)}\left|X^{n}-X^{m}\right|_{\mathcal{L}}=0$. Then there exists $a Y \in \mathcal{L}$ such that $\lim \sup _{N \rightarrow \infty}\left|X^{N}-Y\right|_{\mathcal{L}}=0$.

Proof. Throughout this proof let $N_{k} \in \mathbb{N}, k \in \mathbb{N}$, be a strictly increasing sequence such that for all $k \in \mathbb{N}$ it holds that $\left|X^{N_{k+1}}-X^{N_{k}}\right|_{\mathcal{L}}<\frac{1}{2^{k}}$, let $\mathcal{Y}:[0, T] \times \Omega \rightarrow V_{0}$ be the mapping with the property for all $(t, \omega) \in[0, T] \times \Omega$ it holds that

$$
\mathcal{Y}_{t}(\omega)=\left\{\begin{array}{ll}
\lim _{k \rightarrow \infty} X_{t}^{N_{k}}(\omega) & :\left(X_{t}^{N_{k}}(\omega)\right)_{k \in \mathbb{N}} \text { is convergent in } V_{0} \\
0 & : \text { else }
\end{array},\right.
$$

let $\phi: V_{0} \rightarrow V_{0}$ be the mapping with the property that for all $x \in V_{0}$ it holds that $\phi(x)=\mathbb{1}_{V_{1}}(x)$. $x$, and let $Y:[0, T] \times \Omega \rightarrow V_{0}$ be the mapping with the property for all $(t, \omega) \in[0, T] \times \Omega$ it holds that $Y_{t}(\omega)=\phi\left(\mathbb{1}_{(0, T] \times \Omega}(t, \omega) \cdot \mathcal{Y}_{t}(\omega)\right)+\mathbb{1}_{\{0\} \times \Omega}(t, \omega) \cdot \mathcal{Y}_{0}(\omega)$. The assumption that $\forall N \in \mathbb{N}: X^{N} \in$ 
$\mathcal{M}\left(\operatorname{Pred}\left(\left(\mathcal{F}_{t}\right)_{t \in[0, T]}\right), \mathcal{B}\left(V_{0}\right)\right)$ and, e.g., Exercise 1.74 in Chapter 1 in Hoffmann-Jørgensen [15] imply that $\left\{(t, \omega) \in[0, T] \times \Omega:\left(X_{t}^{N_{k}}(\omega)\right)_{k \in \mathbb{N}}\right.$ is convergent in $\left.V_{0}\right\} \in \operatorname{Pred}\left(\left(\mathcal{F}_{t}\right)_{t \in[0, T]}\right)$. This together with the assumption that $\forall N \in \mathbb{N}: X^{N} \in \mathcal{M}\left(\operatorname{Pred}\left(\left(\mathcal{F}_{t}\right)_{t \in[0, T]}\right), \mathcal{B}\left(V_{0}\right)\right)$ and, e.g., Exercise 1.74 in Chapter 1 in Hoffmann-Jørgensen [15] ensure that $\mathcal{Y} \in \mathcal{M}\left(\operatorname{Pred}\left(\left(\mathcal{F}_{t}\right)_{t \in[0, T]}\right), \mathcal{B}\left(V_{0}\right)\right)$. Furthermore, observe that, e.g., Lemma 2.2 and the fact that

$$
\forall A \in \mathcal{B}\left(V_{0}\right): \phi^{-1}(A)=\phi^{-1}\left(A \cap V_{1}\right)= \begin{cases}A \cap V_{1} & : 0 \notin A \\ \left(V_{0} \backslash V_{1}\right) \cup\left(A \cap V_{1}\right) & : 0 \in A\end{cases}
$$

ensure that $\phi \in \mathcal{M}\left(\mathcal{B}\left(V_{0}\right), \mathcal{B}\left(V_{0}\right)\right)$. Combining this with the fact that $\mathcal{Y} \in \mathcal{M}\left(\operatorname{Pred}\left(\left(\mathcal{F}_{t}\right)_{t \in[0, T]}\right)\right.$, $\left.\mathcal{B}\left(V_{0}\right)\right)$ establishes that $Y \in \mathcal{M}\left(\operatorname{Pred}\left(\left(\mathcal{F}_{t}\right)_{t \in[0, T]}\right), \mathcal{B}\left(V_{0}\right)\right)$ and $Y((0, T] \times \Omega) \subseteq V_{1}$. In the next step we note that the assumption that $\lim _{\sup _{N \rightarrow \infty}} \sup _{n, m \in \mathbb{N} \cap[N, \infty)}\left|X^{n}-X^{m}\right|_{\mathcal{L}}=0$ shows that for all $t \in[0, T]$ it holds that $\lim \sup _{N \rightarrow \infty} \sup _{n, m \in \mathbb{N} \cap[N, \infty)}\left\|X_{t}^{n}-X_{t}^{m}\right\|_{\mathcal{L}^{p}\left(\mathbb{P} ; V_{\mathbb{I}(0, T]}(t)\right)}=0$. Hence, we obtain for every $t \in[0, T]$ that there exists a $\mathfrak{Y}_{t} \in \mathcal{L}^{p}\left(\left.\mathbb{P}\right|_{\mathcal{F}_{t}} ; V_{\mathbb{1}_{(0, T]}(t)}\right)$ such that

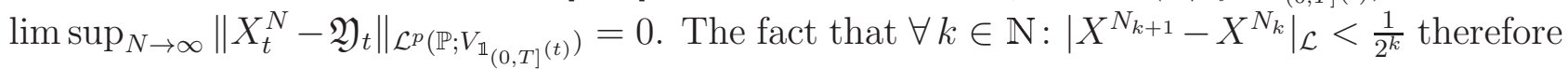
proves that for every $t \in[0, T]$ there exists a $\mathfrak{Y}_{t} \in \mathcal{L}^{p}\left(\mathbb{P}_{\mathcal{F}_{t}} ; V_{\mathbb{1}_{(0, T]}(t)}\right)$ such that for all $n \in \mathbb{N}$ it holds that

$$
\begin{aligned}
& \left.\left\|\mathfrak{Y}_{t}-X_{t}^{N_{n}}\right\|_{\mathcal{L}^{p}\left(\mathbb{P} ; V_{\mathbb{1}}(0, T]\right.}{ }^{(t)}\right) \\
& \left.\left.\leq \limsup _{m \rightarrow \infty}\left(\left\|\mathfrak{Y}_{t}-X_{t}^{N_{m}}\right\|_{\mathcal{L}^{p}\left(\mathbb{P} ; V_{\mathbb{1}}(0, T]\right.}{ }^{(t)}\right)+\left\|X_{t}^{N_{m}}-X_{t}^{N_{n}}\right\|_{\mathcal{L}^{p}\left(\mathbb{P} ; V_{\mathbb{1}}(0, T]\right.}(t)\right)\right) \\
& \left.=\limsup _{m \rightarrow \infty}\left\|\sum_{k=n}^{m-1}\left(X_{t}^{N_{k+1}}-X_{t}^{N_{k}}\right)\right\|_{\mathcal{L}^{p}\left(\mathbb{P} ; V_{\mathbb{I}}(0, T]\right.}(t)\right) \\
& \left.\leq \sum_{k=n}^{\infty}\left\|X_{t}^{N_{k+1}}-X_{t}^{N_{k}}\right\|_{\mathcal{L}^{p}\left(\mathbb{P} ; V_{\mathbb{1}}(0, T]\right.}(t)\right) \\
& \leq \sum_{k=n}^{\infty} t^{-\mathbb{1}_{(0, T]}(t) \cdot \lambda}\left|X_{t}^{N_{k+1}}-X_{t}^{N_{k}}\right|_{\mathcal{L}} \leq t^{-\mathbb{1}_{(0, T]}(t) \cdot \lambda}\left(\sum_{k=n}^{\infty} \frac{1}{2^{k}}\right)=t^{-\mathbb{1}_{(0, T]}(t) \cdot \lambda} 2^{(1-n)} .
\end{aligned}
$$

This and, e.g., item (ii) of Theorem 6.12 in Klenke [19] assure that for every $t \in[0, T]$ there exists a $\mathfrak{Y}_{t} \in \mathcal{L}^{p}\left(\left.\mathbb{P}\right|_{\mathcal{F}_{t}} ; V_{\mathbb{I}_{(0, T]}(t)}\right)$ such that for all $n \in \mathbb{N}$ it holds that $\left\|\mathfrak{Y}_{t}-X_{t}^{N_{n}}\right\|_{\mathcal{L}^{p}\left(\mathbb{P} ; V_{\mathbb{I}_{(0, T]}}(t)\right.} \leq$ $t^{-\mathbb{1}_{(0, T]}(t) \cdot \lambda} 2^{(1-n)}$ and $\mathbb{P}\left(\cap_{k \in \mathbb{N}} \cup_{M \in \mathbb{N}} \cap_{m \in \mathbb{N} \cap[M, \infty)}\left\{\left\|\mathfrak{Y}_{t}-X_{t}^{N_{m}}\right\|_{V_{\mathbb{I}_{(0, T]}(t)}}<\frac{1}{k}\right\}\right)=\mathbb{P}\left(\lim \sup _{m \rightarrow \infty} \| \mathfrak{Y}_{t}-\right.$ $\left.X_{t}^{N_{m}} \|_{V_{\mathbb{I}_{(0, T]}(t)}}=0\right)=1$. The assumption that $V_{1} \subseteq V_{0}$ continuously hence ensures that for all $t \in[0, T], n \in \mathbb{N}$ it holds that $\left.\left\|Y_{t}-X_{t}^{N_{n}}\right\|_{\mathcal{L}^{p}\left(\mathbb{P} ; V_{\mathbb{1}}(0, T]\right.}(t)\right) \leq t^{-\mathbb{1}_{(0, T]}(t) \cdot \lambda} 2^{(1-n)}$. This shows that for all $n \in \mathbb{N}$ it holds that $\left\|Y_{0}-X_{0}^{N_{n}}\right\|_{\mathcal{L}^{p}\left(\mathbb{P} ; V_{0}\right)}+\sup _{t \in(0, T]}\left[t^{\lambda}\left\|Y_{t}-X_{t}^{N_{n}}\right\|_{\mathcal{L}^{p}\left(\mathbb{P} ; V_{1}\right)}\right] \leq 2^{(2-n)}$. Therefore, we get that for all $n \in \mathbb{N}$ it holds that $Y-X^{N_{n}} \in \mathcal{L}$ and $\left|Y-X^{N_{n}}\right|_{\mathcal{L}} \leq 2^{(2-n)}$. Hence, we obtain that $Y \in \mathcal{L}$ and $\lim \sup _{n \rightarrow \infty}\left|Y-X^{N_{n}}\right|_{\mathcal{L}}=0$. This completes the proof of Lemma 2.5.

\subsection{A perturbation estimate for stochastic processes}

Lemma 2.6 is a consequence of the generalized Gronwall inequality from Lemma 7.1.1 in Chapter 7 in Henry [14] (cf. also Exercise 4 in Chapter 7 in Henry [14]). 
Lemma 2.6. Let $\alpha, \beta \in(-\infty, 1), a, b \in[0, \infty), T \in(0, \infty), e \in \mathcal{M}(\mathcal{B}([0, T]), \mathcal{B}([0, \infty]))$ satisfy for all $t \in(0, T]$ that $\int_{0}^{T} e(s) \mathrm{d} s<\infty$ and $e(t) \leq \frac{a}{t^{\alpha}}+\int_{0}^{t} \frac{b e(s)}{(t-s)^{\beta}} \mathrm{d} s$. Then for all $t \in(0, T]$ it holds that $e(t) \leq \frac{a}{t^{\alpha}} \mathrm{E}_{\alpha, \beta}\left[b t^{(1-\beta)}\right]$.

In the next result, Proposition 2.7, we prove a strong perturbation result that will be used several times throughout the paper. We refer to (17) in Subsection 1.1 above for the introduction of the real numbers $\Theta_{A, \eta, p, T}^{\alpha, \beta, \lambda}\left(L_{0}, L_{1}\right)$ appearing on the right hand side of inequality (48) in Proposition 2.7.

Proposition 2.7 (Perturbation estimate). Assume the setting in Section $[$ 2.1, let $\delta \in \mathbb{R}$, and let $Y^{1}, Y^{2}:[0, T] \times \Omega \rightarrow H_{\delta}$ be $\left(\mathcal{F}_{t}\right)_{t \in[0, T]}$-predictable stochastic processes which satisfy $\cup_{k \in\{1,2\}} Y^{k}((0, T] \times \Omega) \subseteq H$ and

$$
\limsup _{\lambda \nearrow \frac{1}{2}\left[1+\mathbb{1}_{\{0\}}\left(L_{1}\right)\right]} \max _{k \in\{1,2\}} \sup _{t \in(0, T]} t^{\lambda}\left\|Y_{t}^{k}\right\|_{L^{p}(\mathbb{P} ; H)}<\infty .
$$

Then

(i) it holds for all $t \in[0, T]$ that

$$
\mathbb{P}\left(\sum_{k=1}^{2} \int_{0}^{t}\left\|e^{(t-s) A} \mathbf{F}\left(s, Y_{s}^{k}\right)\right\|_{H}+\left\|e^{(t-s) A} \mathbf{B}\left(s, Y_{s}^{k}\right)\right\|_{H S(U, H)}^{2} \mathrm{~d} s<\infty\right)=1
$$

and

(ii) it holds for all $\lambda \in\left(-\infty, \frac{1}{2}\left[1+\mathbb{1}_{\{0\}}\left(L_{1}\right)\right]\right)$ that

$$
\begin{aligned}
& \sup _{t \in(0, T]}\left[t^{\lambda}\left\|Y_{t}^{1}-Y_{t}^{2}\right\|_{L^{p}(\mathbb{P} ; H)}\right] \leq \Theta_{A, \eta, p, T}^{\alpha, \beta, \lambda}\left(L_{0}, L_{1}\right) \\
& \cdot \sup _{t \in(0, T]}\left[t^{\lambda} \| Y_{t}^{1}-\int_{0}^{t} e^{(t-s) A} \mathbf{F}\left(s, Y_{s}^{1}\right) \mathrm{d} s-\int_{0}^{t} e^{(t-s) A} \mathbf{B}\left(s, Y_{s}^{1}\right) \mathrm{d} W_{s}\right. \\
& \left.+\int_{0}^{t} e^{(t-s) A} \mathbf{F}\left(s, Y_{s}^{2}\right) \mathrm{d} s+\int_{0}^{t} e^{(t-s) A} \mathbf{B}\left(s, Y_{s}^{2}\right) \mathrm{d} W_{s}-Y_{t}^{2} \|_{L^{p(\mathbb{P} ; H)}}\right] .
\end{aligned}
$$

Proof. Throughout this proof let $r \in\left(-\infty, \frac{1}{2}\left[1+\mathbb{1}_{\{0\}}\left(L_{1}\right)\right]\right)$ and let $\Xi \in[0, \infty]$ be the extended real number given by

$$
\begin{aligned}
& \Xi=\sup _{t \in(0, T]}\left[t^{r} \| Y_{t}^{1}-\int_{0}^{t} e^{(t-s) A} \mathbf{F}\left(s, Y_{s}^{1}\right) \mathrm{d} s-\int_{0}^{t} e^{(t-s) A} \mathbf{B}\left(s, Y_{s}^{1}\right) \mathrm{d} W_{s}\right. \\
& \left.+\int_{0}^{t} e^{(t-s) A} \mathbf{F}\left(s, Y_{s}^{2}\right) \mathrm{d} s+\int_{0}^{t} e^{(t-s) A} \mathbf{B}\left(s, Y_{s}^{2}\right) \mathrm{d} W_{s}-Y_{t}^{2} \|_{L^{p}(\mathbb{P} ; H)}\right] .
\end{aligned}
$$

We observe that item (ii) of Lemma 2.3 and item (ii) of Lemma 2.4 establish that for all $t \in[0, T]$ it holds that $\mathbb{P}\left(\sum_{k=1}^{2} \int_{0}^{t}\left\|e^{(t-s) A} \mathbf{F}\left(s, Y_{s}^{k}\right)\right\|_{H}+\left\|e^{(t-s) A} \mathbf{B}\left(s, Y_{s}^{k}\right)\right\|_{H S(U, H)}^{2} \mathrm{~d} s<\infty\right)=1$. It thus 
remains to prove (48). For this we assume without loss of generality in the following that $\Xi<\infty$. Next we note that the triangle inequality shows that for all $t \in(0, T]$ it holds that

$$
\begin{aligned}
\left\|Y_{t}^{1}-Y_{t}^{2}\right\|_{L^{p}(\mathbb{P} ; H)} \leq & \| Y_{t}^{1}-\int_{0}^{t} e^{(t-s) A} \mathbf{F}\left(s, Y_{s}^{1}\right) \mathrm{d} s-\int_{0}^{t} e^{(t-s) A} \mathbf{B}\left(s, Y_{s}^{1}\right) \mathrm{d} W_{s} \\
& +\int_{0}^{t} e^{(t-s) A} \mathbf{F}\left(s, Y_{s}^{2}\right) \mathrm{d} s+\int_{0}^{t} e^{(t-s) A} \mathbf{B}\left(s, Y_{s}^{2}\right) \mathrm{d} W_{s}-Y_{t}^{2} \|_{L^{p}(\mathbb{P} ; H)} \\
& +\left\|\int_{0}^{t} e^{(t-s) A}\left(\mathbf{F}\left(s, Y_{s}^{1}\right)-\mathbf{F}\left(s, Y_{s}^{2}\right)\right) \mathrm{d} s\right\|_{L^{p}(\mathbb{P} ; H)} \\
& +\left\|\int_{0}^{t} e^{(t-s) A}\left(\mathbf{B}\left(s, Y_{s}^{1}\right)-\mathbf{B}\left(s, Y_{s}^{2}\right)\right) \mathrm{d} W_{s}\right\|_{L^{p}(\mathbb{P} ; H)} .
\end{aligned}
$$

This and the Burkholder-Davis-Gundy type inequality in Lemma 7.7 in Da Prato \& Zabczyk [10] imply that for all $t \in(0, T]$ it holds that

$$
\begin{aligned}
& \left\|Y_{t}^{1}-Y_{t}^{2}\right\|_{L^{p(\mathbb{P} ; H)}} \leq t^{-r} \Xi+\chi_{A, \eta}^{\alpha, T} L_{0} \int_{0}^{t}(t-s)^{-\alpha}\left\|Y_{s}^{1}-Y_{s}^{2}\right\|_{L^{p}(\mathbb{P} ; H)} \mathrm{d} s \\
& +\chi_{A, \eta}^{\beta, T} L_{1}\left[\frac{p(p-1)}{2} \int_{0}^{t}(t-s)^{-2 \beta}\left\|Y_{s}^{1}-Y_{s}^{2}\right\|_{L^{p}(\mathbb{P} ; H)}^{2} \mathrm{~d} s\right]^{1 / 2} .
\end{aligned}
$$

Combining this with Lemma 2.6 proves (48) in the case $L_{1}=0$. It thus remains to prove (48) in the case $L_{1}>0$. For this we observe that (51) together with Hölder's inequality ensures that for all $t \in(0, T]$ it holds that

$$
\begin{aligned}
& \left\|Y_{t}^{1}-Y_{t}^{2}\right\|_{L^{p}(\mathbb{P} ; H)} \leq t^{-r} \Xi \\
& +\chi_{A, \eta}^{\alpha, T} L_{0}\left[T^{\max \{2 \beta-\alpha, 0\}} \int_{0}^{t}(t-s)^{-\alpha} \mathrm{d} s \int_{0}^{t}(t-s)^{-\max \{\alpha, 2 \beta\}}\left\|Y_{s}^{1}-Y_{s}^{2}\right\|_{L^{p}(\mathbb{P} ; H)}^{2} \mathrm{~d} s\right]^{1 / 2} \\
& +\chi_{A, \eta}^{\beta, T} L_{1}\left[\frac{p(p-1)}{2} T^{\max \{\alpha-2 \beta, 0\}} \int_{0}^{t}(t-s)^{-\max \{\alpha, 2 \beta\}}\left\|Y_{s}^{1}-Y_{s}^{2}\right\|_{L^{p}(\mathbb{P} ; H)}^{2} \mathrm{~d} s\right]^{1 / 2} .
\end{aligned}
$$

The fact that $\forall a, b \in \mathbb{R}:(a+b)^{2} \leq 2 a^{2}+2 b^{2}$ hence yields that for all $t \in(0, T]$ it holds that

$$
\begin{aligned}
& \left\|Y_{t}^{1}-Y_{t}^{2}\right\|_{L^{p}(\mathbb{P} ; H)}^{2} \leq \frac{2}{t^{2 r}}|\Xi|^{2}+\int_{0}^{t}(t-s)^{-\max \{\alpha, 2 \beta\}}\left\|Y_{s}^{1}-Y_{s}^{2}\right\|_{L^{p}(\mathbb{P} ; H)}^{2} \mathrm{~d} s \\
& \cdot\left[\chi_{A, \eta}^{\alpha, T} L_{0} \frac{\sqrt{2} T^{1 / 2-\alpha+\max \{\beta, \alpha / 2\}}}{\sqrt{1-\alpha}}+\chi_{A, \eta}^{\beta, T} L_{1} \sqrt{p(p-1)} T^{\max \{\alpha / 2, \beta\}-\beta}\right]^{2} .
\end{aligned}
$$

Combining this with Lemma 2.6 and the fact that

$$
\begin{aligned}
& E_{2 r, \max \{\alpha, 2 \beta\}}\left[T^{(1-\max \{\alpha, 2 \beta\})}\left|\chi_{A, \eta}^{\alpha, T} L_{0} \frac{\sqrt{2} T^{1 / 2-\alpha+\max \{\beta, \alpha / 2\}}}{\sqrt{1-\alpha}}+\chi_{A, \eta}^{\beta, T} L_{1} \sqrt{p(p-1)} T^{\max \{\alpha / 2, \beta\}-\beta}\right|^{2}\right] \\
& =E_{2 r, \max \{\alpha, 2 \beta\}}\left[\left|\chi_{A, \eta}^{\alpha, T} L_{0} \frac{\sqrt{2} T^{(1-\alpha)}}{\sqrt{1-\alpha}}+\chi_{A, \eta}^{\beta, T} L_{1} \sqrt{p(p-1) T^{(1-2 \beta)}}\right|^{2}\right]
\end{aligned}
$$


ensures that for all $t \in(0, T]$ it holds that

$$
\begin{aligned}
& \left\|Y_{t}^{1}-Y_{t}^{2}\right\|_{L^{p}(\mathbb{P} ; H)}^{2} \\
& \leq \frac{2}{t^{2 r}}|\Xi|^{2} E_{2 r, \max \{\alpha, 2 \beta\}}\left[\left|\chi_{A, \eta}^{\alpha, T} L_{0} \frac{\sqrt{2} T^{(1-\alpha)}}{\sqrt{1-\alpha}}+\chi_{A, \eta}^{\beta, T} L_{1} \sqrt{p(p-1) T^{(1-2 \beta)}}\right|^{2}\right] .
\end{aligned}
$$

Hence, we obtain that

$$
\begin{aligned}
& \sup _{t \in(0, T]}\left[t^{r}\left\|Y_{t}^{1}-Y_{t}^{2}\right\|_{L^{p}(\mathbb{P} ; H)}\right] \\
& \leq \sqrt{2} \Xi\left|E_{2 r, \max \{\alpha, 2 \beta\}}\left[\left|\chi_{A, \eta}^{\alpha, T} L_{0} \frac{\sqrt{2} T^{(1-\alpha)}}{\sqrt{1-\alpha}}+\chi_{A, \eta}^{\beta, T} L_{1} \sqrt{p(p-1) T^{(1-2 \beta)}}\right|^{2}\right]\right|^{1 / 2} .
\end{aligned}
$$

This finishes the proof of Proposition 2.7.

In the next result, Corollary 2.8, we illustrate Proposition 2.5 by a simple example. In particular, Corollary 2.8 ensures uniqueness of solutions of SEEs with singularities at the initial time. We refer, e.g., to item (i) of Theorem 7.4 in Da Prato \& Zabczyk [10] for an existence and uniqueness result for SEEs without singularities at the initial time.

Corollary 2.8 (Initial conditions). Assume the setting in Section [2.1, let $\delta \in\left[0, \frac{1}{2}\left[1+\mathbb{1}_{\{0\}}\left(L_{1}\right)\right]\right)$, and let $X^{1}, X^{2}:[0, T] \times \Omega \rightarrow H_{-\delta}$ be $\left(\mathcal{F}_{t}\right)_{t \in[0, T]}$-predictable stochastic processes which fulfill for all $k \in\{1,2\}, t \in[0, T]$ that $X^{k}((0, T] \times \Omega) \subseteq H$, that $\lim \sup _{\lambda \nearrow \frac{1}{2}\left[1+\mathbb{1}_{\{0\}}\left(L_{1}\right)\right]} \sup _{s \in(0, T]} s^{\lambda}\left\|X_{s}^{k}\right\|_{L^{p}(\mathbb{P} ; H)}$ $<\infty$, and $\mathbb{P}$-a.s. that

$$
X_{t}^{k}=e^{t A} X_{0}^{k}+\int_{0}^{t} e^{(t-s) A} \mathbf{F}\left(s, X_{s}^{k}\right) \mathrm{d} s+\int_{0}^{t} e^{(t-s) A} \mathbf{B}\left(s, X_{s}^{k}\right) \mathrm{d} W_{s} .
$$

Then it holds for all $\lambda \in\left[\delta, \frac{1}{2}\left[1+\mathbb{1}_{\{0\}}\left(L_{1}\right)\right]\right)$ that

$$
\sup _{t \in(0, T]}\left[t^{\lambda}\left\|X_{t}^{1}-X_{t}^{2}\right\|_{L^{p}(\mathbb{P} ; H)}\right] \leq \chi_{A, \eta}^{\delta, T} T^{(\lambda-\delta)}\left\|X_{0}^{1}-X_{0}^{2}\right\|_{L^{p}\left(\mathbb{P} ; H_{-\delta}\right)} \Theta_{A, \eta, p, T}^{\alpha, \beta, \lambda}\left(L_{0}, L_{1}\right) .
$$

\subsection{Existence, uniqueness, and regularity for SEEs with singularities at the initial time}

In Theorem 2.9 below we establish existence, uniqueness, and regularity for SEEs with singularities at the initial time. The following remark helps to access the formulation of Theorem 2.9.

Remark. Assume the setting in Section 2.1 and let $\delta \in\left(-\infty, \frac{1}{2}\left[1+\mathbb{1}_{\{0\}}\left(L_{1}\right)\right]\right)$. Observe that the assumptions that $\alpha<1, \hat{\alpha}<1, \beta<1 / 2, \hat{\beta}<1 / 2$, and $\mathbb{1}_{(0, \infty)}\left(L_{1}\right) \cdot[\alpha+\hat{\alpha}]<3 / 2$ ensure that

$$
\max \{\delta, \alpha+\hat{\alpha}-1, \beta+\hat{\beta}-1 / 2\}<\frac{1}{2}\left[1+\mathbb{1}_{\{0\}}\left(L_{1}\right)\right] .
$$

We now present the promised existence, uniqueness, and regularity results for SEEs with singularities at the initial time. 
Theorem 2.9. Assume the setting in Section [2.1 and let $\delta \in\left(-\infty, \frac{1}{2}\left[1+\mathbb{1}_{\{0\}}\left(L_{1}\right)\right]\right), \lambda \in$ $\left[\max \{\delta, \alpha+\hat{\alpha}-1, \beta+\hat{\beta}-1 / 2\}, \frac{1}{2}\left[1+\mathbb{1}_{\{0\}}\left(L_{1}\right)\right]\right), \rho=\max \left\{\lambda+(\hat{\beta}-\lambda) \mathbb{1}_{\{0\}}\left(L_{1}\right), \hat{\beta}\right\}, \xi \in$ $\mathcal{L}^{p}\left(\left.\mathbb{P}\right|_{\mathcal{F}_{0}} ; H_{-\max \{\delta, 0\}}\right)$ satisfy $\sup _{t \in(0, T]} t^{\delta}\left\|e^{t A} \xi\right\|_{L^{p}(\mathbb{P} ; H)}<\infty$. Then

(i) there exists an up-to-modifications unique $\left(\mathcal{F}_{t}\right)_{t \in[0, T]}$-predictable stochastic process $X:[0, T] \times$ $\Omega \rightarrow H_{-\max \{\delta, 0\}}$ which satisfies for all $t \in[0, T]$ that $X((0, T] \times \Omega) \subseteq H$, that $\sup _{s \in(0, T]} s^{\lambda}$ $\left\|X_{s}\right\|_{L^{p}(\mathbb{P} ; H)}<\infty$, that $\mathbb{P}\left(\int_{0}^{t}\left\|e^{(t-s) A} \mathbf{F}\left(s, X_{s}\right)\right\|_{H}+\left\|e^{(t-s) A} \mathbf{B}\left(s, X_{s}\right)\right\|_{H S(U, H)}^{2} \mathrm{~d} s<\infty\right)=1$, and $\mathbb{P}$-a.s. that

$$
X_{t}=e^{t A} \xi+\int_{0}^{t} e^{(t-s) A} \mathbf{F}\left(s, X_{s}\right) \mathrm{d} s+\int_{0}^{t} e^{(t-s) A} \mathbf{B}\left(s, X_{s}\right) \mathrm{d} W_{s},
$$

(ii) it holds that

$$
\begin{aligned}
& \sup _{t \in(0, T]}\left[t^{\lambda}\left\|X_{t}\right\|_{L^{p}(\mathbb{P} ; H)}\right] \leq T^{\lambda} \Theta_{A, \eta, p, T}^{\alpha, \beta, \lambda}\left(L_{0}, L_{1}\right) \\
& \quad \cdot\left[\frac{\sup _{t \in(0, T]}\left(t^{\delta}\left\|e^{t A} \xi\right\|_{L^{p}(\mathbb{P} ; H)}\right)}{T^{\delta}}+\frac{\chi_{A, \eta}^{\alpha, T} \hat{L}_{0} \mathbb{B}(1-\alpha, 1-\hat{\alpha})}{T^{(\alpha+\hat{\alpha}-1)}}+\frac{\chi_{A, \eta}^{\beta, T} \hat{L}_{1}|p(p-1) \mathbb{B}(1-2 \beta, 1-2 \hat{\beta})|^{1 / 2}}{\sqrt{2} T^{(\beta+\hat{\beta}-1 / 2)}}\right]<\infty,
\end{aligned}
$$

(iii) and for all $\varrho \in[0, \min \{1-\alpha, 1 / 2-\beta\}), s, t \in(0, T]$ with $s<t$ it holds that

$$
\begin{aligned}
& \left\|X_{s}-X_{t}\right\|_{L^{p(\mathbb{P} ; H)}} \leq|s-t|^{\varrho} \\
& \cdot\left[\frac{\kappa_{A, \eta}^{\varrho, T} \chi_{A, \eta}^{\varrho+\max \{\delta, 0\}, T}\|\xi\|_{L^{p}\left(\mathbb{P} ; H_{-} \max \{\delta, 0\}\right)}}{s^{(\varrho+\max \{\delta, 0\})}}+|T \vee 1|^{|\lambda-\hat{\alpha}|}\left(\hat{L}_{0}+L_{0} \sup _{u \in(0, T]} u^{\lambda}\left\|X_{u}\right\|_{L^{p}(\mathbb{P} ; H)}\right)\right. \\
& \cdot\left[\frac{\chi_{A, \eta}^{\alpha, T}|s-t|^{(1-\alpha-\varrho)}}{(1-\alpha) \min \left\{s^{\left.\max \{\lambda, \hat{\alpha}\}, t^{\max }\{\lambda, \hat{\alpha}\}\right\}}\right.}+\frac{\kappa_{A, \eta}^{\varrho, T} \chi_{A, \eta}^{\varrho+\alpha, T} \mathbb{B}(1-\alpha-\varrho, 1-\max \{\lambda, \hat{\alpha}\})}{s^{(\varrho+\alpha+\max \{\lambda, \hat{\alpha}\}-1)}}\right] \\
& +\sqrt{\frac{p(p-1)}{2}}|T \vee 1|^{|\lambda-\hat{\beta}| \mathbb{1}_{(0, \infty)}\left(L_{1}\right)}\left(\hat{L}_{1}+L_{1} \sup _{u \in(0, T]} u^{\lambda}\left\|X_{u}\right\|_{L^{p}(\mathbb{P} ; H)}\right) \\
& \left.\cdot\left[\frac{\chi_{A, \eta}^{\beta, T}|s-t|^{(1 / 2-\beta-\varrho)}}{\min \left\{s^{\rho}, t^{\rho}\right\} \sqrt{1-2 \beta}}+\frac{\kappa_{A, \eta}^{\varrho, T} \chi_{A, \eta}^{\varrho+\beta, T}|\mathbb{B}(1-2 \beta-2 \varrho, 1-2 \rho)|^{1 / 2}}{s^{(\rho+\varrho+\beta-1 / 2)}}\right]\right] \text {. }
\end{aligned}
$$

Proof. Throughout this proof let $\mathcal{L}$ and $\mathbb{L}$ be the sets given by

$$
\mathcal{L}=\left\{\begin{array}{c}
X \in \mathcal{M}\left(\operatorname{Pred}\left(\left(\mathcal{F}_{t}\right)_{t \in[0, T]}\right), \mathcal{B}\left(H_{-\max \{\delta, 0\}}\right)\right): X((0, T] \times \Omega) \subseteq H, \\
\left\|X_{0}\right\|_{L^{p}(\mathbb{P} ; H}-\max \{\delta, 0\} \\
)+\sup _{t \in(0, T]} t^{\lambda}\left\|X_{t}\right\|_{L^{p}(\mathbb{P} ; H)}<\infty
\end{array}\right\}
$$

and $\mathbb{L}=\left\{\left\{Y \in \mathcal{L}: \inf _{t \in[0, T]} \mathbb{P}\left(Y_{t}=X_{t}\right)=1\right\} \subseteq \mathcal{L}: X \in \mathcal{L}\right\}$ and let $|\cdot|_{\mathbb{L}, r}: \mathbb{L} \rightarrow[0, \infty), r \in \mathbb{R}$, and $\|\cdot\|_{\mathbb{L}, r}: \mathbb{L} \rightarrow[0, \infty), r \in \mathbb{R}$, be the functions which satisfy for all $r \in \mathbb{R}, X \in \mathbb{L}$ that

$$
|X|_{\mathbb{L}, r}=\sup _{t \in(0, T]}\left[e^{r t} t^{\lambda}\left\|X_{t}\right\|_{L^{p}(\mathbb{P} ; H)}\right] \quad \text { and } \quad\|X\|_{\mathbb{L}, r}=\left\|X_{0}\right\|_{L^{p}\left(\mathbb{P} ; H_{-\max \{\delta, 0\}}\right)}+|X|_{\mathbb{L}, r}
$$

Here and below we do not distinguish between an element $X \in \mathcal{L}$ and its equivalence class $\left\{Y \in \mathcal{L}: \inf _{t \in[0, T]} \mathbb{P}\left(Y_{t}=X_{t}\right)=1\right\} \in \mathbb{L}$. We observe that for all $t \in(0, T]$ it holds that

$$
t^{\lambda}\left\|e^{t A} \xi\right\|_{L^{p}(\mathbb{P} ; H)} \leq T^{(\lambda-\delta)} \sup _{s \in(0, T]} s^{\delta}\left\|e^{s A} \xi\right\|_{L^{p}(\mathbb{P} ; H)}<\infty .
$$


This ensures that

$$
\left([0, T] \times \Omega \ni(t, \omega) \mapsto e^{t A} \xi(\omega) \in H_{-\max \{\delta, 0\}}\right) \in \mathcal{L} .
$$

Combining this with Lemma 2.3 and Lemma 2.4 shows that there exists a unique mapping $\Phi: \mathbb{L} \rightarrow \mathbb{L}$ which satisfies that for all $Y \in \mathbb{L}, t \in[0, T]$ it holds $\mathbb{P}$-a.s. that

$$
\Phi(Y)_{t}=e^{t A} \xi+\int_{0}^{t} e^{(t-s) A} \mathbf{F}\left(s, Y_{s}\right) \mathrm{d} s+\int_{0}^{t} e^{(t-s) A} \mathbf{B}\left(s, Y_{s}\right) \mathrm{d} W_{s} .
$$

Our next aim is to prove that there exists a real number $r \in \mathbb{R}$ such that $\Phi$ is a contraction on the normed $\mathbb{R}$-vector space $\left(\mathbb{L},\|\cdot\|_{\mathbb{L}, r}\right)$. Banach's fixed point theorem together with Lemma 2.5 will then allow us to prove (iil). Observe that the Burkholder-Davis-Gundy type inequality in Lemma 7.7 in Da Prato \& Zabczyk [10] proves that for all $Y, Z \in \mathbb{L}, r \in \mathbb{R}, t \in[0, T]$ it holds that

$$
\begin{aligned}
\| & \Phi(Y)_{t}-\Phi(Z)_{t}\left\|_{L^{p}(\mathbb{P} ; H)} \leq\right\| \int_{0}^{t} e^{(t-s) A}\left(\mathbf{F}\left(s, Y_{s}\right)-\mathbf{F}\left(s, Z_{s}\right)\right) \mathrm{d} s \|_{L^{p}(\mathbb{P} ; H)} \\
& +\left\|\int_{0}^{t} e^{(t-s) A}\left(\mathbf{B}\left(s, Y_{s}\right)-\mathbf{B}\left(s, Z_{s}\right)\right) \mathrm{d} W_{s}\right\|_{L^{p}(\mathbb{P} ; H)} \\
\leq & \int_{0}^{t}\left\|e^{(t-s) A}\right\|_{L\left(H_{-\alpha}, H\right)}\left\|\mathbf{F}\left(Y_{s}\right)-\mathbf{F}\left(Z_{s}\right)\right\|_{L^{p}\left(\mathbb{P} ; H_{-\alpha}\right)} \mathrm{d} s \\
& +\left[\frac{p(p-1)}{2} \int_{0}^{t}\left\|e^{(t-s) A}\right\|_{L\left(H_{-\beta}, H\right)}^{2}\left\|\mathbf{B}\left(Y_{s}\right)-\mathbf{B}\left(Z_{s}\right)\right\|_{L^{p}\left(\mathbb{P} ; H S\left(U, H_{-\beta}\right)\right)}^{2} \mathrm{~d} s\right]^{1 / 2} \\
\leq & \chi_{A, \eta}^{\alpha, T} \int_{0}^{t} L_{0}(t-s)^{-\alpha}\left\|Y_{s}-Z_{s}\right\|_{L^{p}(\mathbb{P} ; H)} \mathrm{d} s \\
& +\chi_{A, \eta}^{\beta, T}\left[\frac{p(p-1)}{2} \int_{0}^{t}\left|L_{1}\right|^{2}(t-s)^{-2 \beta}\left\|Y_{s}-Z_{s}\right\|_{L^{p}(\mathbb{P} ; H)}^{2} \mathrm{~d} s\right]^{\frac{1}{2}} \\
\leq & \chi_{A, \eta}^{\alpha, T}|Y-Z|_{\mathbb{L}, r} \int_{0}^{t} L_{0}(t-s)^{-\alpha} s^{-\lambda} e^{-r s} \mathrm{~d} s \\
& +\chi_{A, T}^{\beta, T}|Y-Z|_{\mathbb{L}, r}\left[\frac{p(p-1)}{2} \int_{0}^{t}\left|L_{1}\right|^{2}(t-s)^{-2 \beta} s^{-2 \lambda} e^{-2 r s} \mathrm{~d} s\right]^{\frac{1}{2}} \\
\leq & {\left[\chi_{A, \eta}^{\alpha, T} \int_{0}^{t} L_{0} e^{-r s}(t-s)^{-\alpha} s^{-\lambda} \mathrm{d} s+\chi_{A, T}^{\beta, T}\left[\frac{p(p-1)}{2} \int_{0}^{t}\left|L_{1}\right|^{2} e^{-2 r s}(t-s)^{-2 \beta} s^{-2 \lambda} \mathrm{d} s\right]^{\frac{1}{2}}\right] } \\
& \cdot|Y-Z|_{\mathbb{L}, r}<\infty .
\end{aligned}
$$

Hence, we obtain that for all $Y, Z \in \mathbb{L}, r \in(-\infty, 0]$ it holds that

$$
\begin{aligned}
& \|\Phi(Y)-\Phi(Z)\|_{\mathbb{L}, r} \\
& =\left\|\Phi(Y)_{0}-\Phi(Z)_{0}\right\|_{L^{p}\left(\mathbb{P} ; H_{-\max \{\delta, 0\}}\right)}+\sup _{t \in(0, T]}\left[e^{r t} t^{\lambda}\left\|\Phi(Y)_{t}-\Phi(Z)_{t}\right\|_{L^{p}(\mathbb{P} ; H)}\right] \\
& \leq \sup _{t \in(0, T]}\left[\chi_{A, \eta}^{\alpha, T} \int_{0}^{t} \frac{L_{0} e^{r(t-s)} t^{\lambda}}{(t-s)^{\alpha} s^{\lambda}} \mathrm{d} s+\chi_{A, T}^{\beta, T}\left[\frac{p(p-1)}{2} \int_{0}^{t} \frac{\left|L_{1}\right|^{2} e^{2 r(t-s)} t^{2 \lambda}}{(t-s)^{2 \beta} s^{2 \lambda}} \mathrm{d} s\right]^{\frac{1}{2}}\right]|Y-Z|_{\mathbb{L}, r} .
\end{aligned}
$$


This and the integral transformation theorem with the diffeomorphisms $(0,1) \ni s \mapsto t(1-s) \in$ $(0, t)$ for $t \in(0, T]$ show that for all $Y, Z \in \mathbb{L}, r \in(-\infty, 0]$ it holds that

$$
\begin{aligned}
& \|\Phi(Y)-\Phi(Z)\|_{\mathbb{L}, r} \leq|Y-Z|_{\mathbb{L}, r} \\
& \cdot\left(\chi_{A, \eta}^{\alpha, T} \sup _{t \in(0, T]}\left[\int_{0}^{1} \frac{L_{0} e^{r t s} t^{(1-\alpha)}}{s^{\alpha}(1-s)^{\lambda}} \mathrm{d} s\right]+\chi_{A, T}^{\beta, T}\left[\frac{p(p-1)}{2} \sup _{t \in(0, T]}\left[\int_{0}^{1} \frac{\left|L_{1}\right|^{2} e^{2 r t s} t^{(1-2 \beta)}}{s^{2 \beta}(1-s)^{2 \lambda}} \mathrm{d} s\right]\right]^{\frac{1}{2}}\right) .
\end{aligned}
$$

Next note that Lebesgue's theorem of dominated convergence ensures that for all $r \in \mathbb{R}$ it holds that the functions

$$
[0, T] \ni t \mapsto \int_{0}^{1} \frac{L_{0} e^{r t s} t^{(1-\alpha)}}{s^{\alpha}(1-s)^{\lambda}} \mathrm{d} s=L_{0} t^{(1-\alpha)} \int_{0}^{1} \frac{e^{r t s}}{s^{\alpha}(1-s)^{\lambda}} \mathrm{d} s \in[0, \infty)
$$

and

$$
[0, T] \ni t \mapsto \int_{0}^{1} \frac{\left|L_{1}\right|^{2} e^{2 r t s} t^{(1-2 \beta)}}{s^{2 \beta}(1-s)^{2 \lambda}} \mathrm{d} s=t^{(1-2 \beta)} \int_{0}^{1} \frac{\left|L_{1}\right|^{2} e^{2 r t s}}{s^{2 \beta}(1-s)^{2 \lambda}} \mathrm{d} s \in[0, \infty)
$$

are continuous. This and the fact that for all $t \in[0, T]$ it holds that

$$
\limsup _{r \rightarrow-\infty}\left[\int_{0}^{1} \frac{L_{0} e^{r t s} t^{(1-\alpha)}}{s^{\alpha}(1-s)^{\lambda}} \mathrm{d} s\right]=\limsup _{r \rightarrow-\infty}\left[\int_{0}^{1} \frac{\left|L_{1}\right|^{2} e^{2 r t s} t^{(1-2 \beta)}}{s^{2 \beta}(1-s)^{2 \lambda}} \mathrm{d} s\right]=0
$$

allows us to apply Dini's theorem (see, e.g., Theorem 7.13 in Rudin [25]) to obtain that

$$
\begin{aligned}
& \limsup _{r \rightarrow-\infty}\left(\chi_{A, \eta}^{\alpha, T} \sup _{t \in(0, T]}\left[\int_{0}^{1} \frac{L_{0} e^{r t s} t^{(1-\alpha)}}{s^{\alpha}(1-s)^{\lambda}} \mathrm{d} s\right]+\chi_{A, T}^{\beta, T}\left[\frac{p(p-1)}{2} \sup _{t \in(0, T]}\left[\int_{0}^{1} \frac{\left|L_{1}\right|^{2} e^{2 r t s} t^{(1-2 \beta)}}{s^{2 \beta}(1-s)^{2 \lambda}} \mathrm{d} s\right]\right]^{\frac{1}{2}}\right) \\
& =0 .
\end{aligned}
$$

The Banach fixed point theorem together with Lemma 2.5 and (69) hence establishes (1i), that is, there exists an up-to-modifications unique $X \in \mathcal{L}$ which fulfills that for all $t \in[0, T]$ it holds that $\mathbb{P}\left(\int_{0}^{t}\left\|e^{(t-s) A} \mathbf{F}\left(s, X_{s}\right)\right\|_{H}+\left\|e^{(t-s) A} \mathbf{B}\left(s, X_{s}\right)\right\|_{H S(U, H)}^{2} \mathrm{~d} s<\infty\right)=1$ and (60) $)$. In the next step we observe that (iii) follows directly from item (iv) of Lemma 2.3, from item (iv) of Lemma [2.4, and from the fact that $\forall \varrho \in[0,1], t \in(0, T], s \in(0, t):\left\|e^{t A} \xi-e^{s A} \xi\right\|_{L^{p}(\mathbb{P} ; H)} \leq$ $\frac{|t-s|^{\varrho}}{s^{(\varrho+\max \{\delta, 0\})}} \kappa_{A, \eta}^{\varrho, T} \chi_{A, \eta}^{\varrho+\max \{\delta, 0\}, T}\|\xi\|_{L^{p}\left(\mathbb{P} ; H_{-\max \{\delta, 0\}}\right)}$. It thus remains to prove (iii). For this we apply Proposition 2.7 (with $Y^{1}=X, Y^{2}=0$, and $r=\lambda$ in the notation of Proposition 2.7) to obtain that

$$
\begin{aligned}
& \sup _{t \in(0, T]}\left[t^{\lambda}\left\|X_{t}\right\|_{L^{p}(\Omega ; H)}\right] \leq \Theta_{A, \eta, p, T}^{\alpha, \beta, \lambda}\left(L_{0}, L_{1}\right) \\
& \cdot \sup _{t \in(0, T]}\left[t^{\lambda} \| X_{t}-\int_{0}^{t} e^{(t-s) A} \mathbf{F}\left(s, X_{s}\right) \mathrm{d} s-\int_{0}^{t} e^{(t-s) A} \mathbf{B}\left(s, X_{s}\right) \mathrm{d} W_{s}\right. \\
& \left.+\int_{0}^{t} e^{(t-s) A} \mathbf{F}(s, 0) \mathrm{d} s+\int_{0}^{t} e^{(t-s) A} \mathbf{B}(s, 0) \mathrm{d} W_{s} \|_{L^{p}(\mathbb{P} ; H)}\right] \\
& =\Theta_{A, \eta, p, T}^{\alpha, \beta, \lambda}\left(L_{0}, L_{1}\right) \\
& \text {. } \sup _{t \in(0, T]}\left[t^{\lambda}\left\|e^{t A} \xi+\int_{0}^{t} e^{(t-s) A} \mathbf{F}(s, 0) \mathrm{d} s+\int_{0}^{t} e^{(t-s) A} \mathbf{B}(s, 0) \mathrm{d} W_{s}\right\|_{L^{p}(\mathbb{P} ; H)}\right] \text {. }
\end{aligned}
$$


Next we note that the Burkholder-Davis-Gundy type inequality in Lemma 7.7 in Da Prato \& Zabczyk [10] implies that for all $t \in[0, T]$ it holds that

$$
\begin{aligned}
& \left\|e^{t A} \xi+\int_{0}^{t} e^{(t-s) A} \mathbf{F}(s, 0) \mathrm{d} s+\int_{0}^{t} e^{(t-s) A} \mathbf{B}(s, 0) \mathrm{d} W_{s}\right\|_{L^{p}(\mathbb{P} ; H)} \\
& \leq\left\|e^{t A} \xi\right\|_{L^{p}(\mathbb{P} ; H)}+\int_{0}^{t}\left\|e^{(t-s) A} \mathbf{F}(s, 0)\right\|_{L^{p}(\mathbb{P} ; H)} \mathrm{d} s+\left[\frac{p(p-1)}{2} \int_{0}^{t}\left\|e^{(t-s) A} \mathbf{B}(s, 0)\right\|_{L^{p}(\mathbb{P} ; H)}^{2} \mathrm{~d} s\right]^{1 / 2} \\
& \leq\left\|e^{t A} \xi\right\|_{L^{p}(\mathbb{P} ; H)}+\chi_{A, \eta}^{\alpha, T} \hat{L}_{0} \int_{0}^{t}(t-s)^{-\alpha} s^{-\hat{\alpha}} \mathrm{d} s+\chi_{A, \eta}^{\beta, T} \hat{L}_{1}\left[\frac{p(p-1)}{2} \int_{0}^{t}(t-s)^{-2 \beta} s^{-2 \hat{\beta}} \mathrm{d} s\right]^{1 / 2} \\
& \leq\left\|e^{t A} \xi\right\|_{L^{p(\mathbb{P} ; H)}}+\frac{\chi_{A, \eta}^{\alpha, T} \hat{L}_{0} \mathbb{B}(1-\alpha, 1-\hat{\alpha})}{t^{(\alpha+\hat{\alpha}-1)}}+\frac{\chi_{A, \eta}^{\beta, T} \hat{L}_{1} \sqrt{p(p-1) \mathbb{B}(1-2 \beta, 1-2 \hat{\beta})}}{\sqrt{2} t^{(\beta+\hat{\beta}-1 / 2)}} .
\end{aligned}
$$

This shows that

$$
\begin{aligned}
& \sup _{t \in(0, T]}\left[t^{\lambda}\left\|e^{t A} \xi+\int_{0}^{t} e^{(t-s) A} \mathbf{F}(s, 0) \mathrm{d} s+\int_{0}^{t} e^{(t-s) A} \mathbf{B}(s, 0) \mathrm{d} W_{s}\right\|_{L^{p}(\mathbb{P} ; H)}\right] \\
& \leq T^{(\lambda-\delta)} \sup _{t \in(0, T]}\left[t^{\delta}\left\|e^{t A} \xi\right\|_{L^{p}(\mathbb{P} ; H)}\right]+\chi_{A, \eta}^{\alpha, T} \hat{L}_{0} T^{(\lambda+1-\alpha-\hat{\alpha})} \mathbb{B}(1-\alpha, 1-\hat{\alpha}) \\
& \quad+\frac{\chi_{A, \eta}^{\beta, T} \hat{L}_{1} T^{(\lambda+1 / 2-\beta-\hat{\beta})} \sqrt{p(p-1) \mathbb{B}(1-2 \beta, 1-2 \hat{\beta})}}{\sqrt{2}}<\infty .
\end{aligned}
$$

Combining this with (75) proves (iii). The proof of Theorem 2.9 is thus completed.

Corollary 2.10. Let $\left(H,\|\cdot\|_{H},\langle\cdot, \cdot\rangle_{H}\right)$ and $\left(U,\|\cdot\|_{U},\langle\cdot, \cdot\rangle_{U}\right)$ be separable $\mathbb{R}$-Hilbert spaces with $\#_{H}(H)>1$, let $T \in(0, \infty), \eta \in \mathbb{R}, \alpha \in[0,1), \beta \in[0,1 / 2)$, let $\left(\Omega, \mathcal{F}, \mathbb{P},\left(\mathcal{F}_{t}\right)_{t \in[0, T]}\right)$ be a stochastic basis, let $\left(W_{t}\right)_{t \in[0, T]}$ be an $\operatorname{Id}_{U}$-cylindrical $\left(\mathcal{F}_{t}\right)_{t \in[0, T]}$-Wiener process, let $A: D(A) \subseteq$ $H \rightarrow H$ be a generator of a strongly continuous analytic semigroup with the property that $\operatorname{spectrum}(A) \subseteq\{z \in \mathbb{C}: \operatorname{Re}(z)<\eta\}$, let $\left(H_{r},\|\cdot\|_{H_{r}},\langle\cdot, \cdot\rangle_{H_{r}}\right), r \in \mathbb{R}$, be a family of interpolation spaces associated to $\eta-A$, and let $F \in \operatorname{Lip}\left(H, H_{-\alpha}\right), B \in \operatorname{Lip}\left(H, H S\left(U, H_{-\beta}\right)\right), \hat{\delta}=\frac{1}{2}[1+$ $\left.\mathbb{1}_{\{0\}}\left(|B|_{\operatorname{Lip}\left(H, H S\left(U, H_{-\beta}\right)\right)}\right)\right]$. Then

(i) there exist up-to-modifications unique $\left(\mathcal{F}_{t}\right)_{t \in[0, T]}$-predictable stochastic processes $X^{x}:[0, T] \times$ $\Omega \rightarrow H_{-\delta}, x \in H_{-\delta}, \delta \in[0, \hat{\delta})$, which fulfill for all $p \in[2, \infty), \delta \in[0, \hat{\delta}), x \in H_{-\delta}, t \in[0, T]$ that $X^{x}((0, T] \times \Omega) \subseteq H$, that $\sup _{s \in(0, T]} s^{\delta}\left\|X_{s}^{x}\right\|_{L^{p}(\mathbb{P} ; H)}<\infty$, and $\mathbb{P}$-a.s. that

$$
X_{t}^{x}=e^{t A} x+\int_{0}^{t} e^{(t-s) A} F\left(X_{s}^{x}\right) \mathrm{d} s+\int_{0}^{t} e^{(t-s) A} B\left(X_{s}^{x}\right) \mathrm{d} W_{s},
$$

(ii) for all $p \in[2, \infty), \delta \in[0, \hat{\delta})$ it holds that

$$
\begin{aligned}
& \sup _{x \in H_{-\delta}} \sup _{t \in(0, T]}\left[\frac{t^{\delta}\left\|X_{t}^{x}\right\|_{L^{p}(\mathbb{P} ; H)}}{\max \left\{1,\|x\|_{H_{-\delta}}\right\}}\right] \leq \Theta_{A, \eta, p, T}^{\alpha, \beta, \delta}\left(|F|_{\operatorname{Lip}\left(H, H_{-\alpha}\right)},|B|_{\operatorname{Lip}\left(H, H S\left(U, H_{-\beta}\right)\right)}\right) \\
& \cdot\left[\chi_{A, \eta}^{\delta, T}+\frac{\chi_{A, \eta}^{\alpha, T}\|F(0)\|_{H_{-\alpha}} T^{(\delta+1-\alpha)}}{(1-\alpha)}+\frac{\sqrt{p(p-1)} \chi_{A, \eta}^{\beta, T}\|B(0)\|_{H S(U, H-\beta} T^{(\delta+1 / 2-\beta)}}{\sqrt{2-4 \beta}}\right]<\infty,
\end{aligned}
$$


(iii) for all $p \in[2, \infty), \delta \in[0, \hat{\delta})$ it holds that

$$
\begin{aligned}
& \sup _{\substack{x, y \in H_{-\delta}, t \in(0, T] \\
x \neq y}} \sup _{t \in T^{\prime}}\left[\frac{t^{\delta}\left\|X_{t}^{x}-X_{t}^{y}\right\|_{L^{p}(\mathbb{P} ; H)}}{\|x-y\|_{H_{-\delta}}}\right] \\
& \leq \chi_{A, \eta}^{\delta, T} \Theta_{A, \eta, p, T}^{\alpha, \beta, \delta}\left(|F|_{\operatorname{Lip}\left(H, H_{-\alpha}\right)},|B|_{\operatorname{Lip}\left(H, H S\left(U, H_{-\beta}\right)\right)}\right)<\infty,
\end{aligned}
$$

(iv) and for all $p \in[2, \infty), \delta \in[0, \hat{\delta}), \varrho \in[0, \min \{1-\alpha, 1 / 2-\beta\})$ it holds that

$$
\sup _{t \in(0, T]} \sup _{s \in(0, t)} \sup _{x \in H_{-\delta}}\left[\frac{s^{(\varrho+\delta)}\left\|X_{s}^{x}-X_{t}^{x}\right\|_{L^{p}(\mathbb{P} ; H)}}{\max \left\{1,\|x\|_{H_{-\delta}}\right\}|s-t| \varrho}\right]<\infty .
$$

Proof of Corollary 2.10. Throughout this proof let $L_{0}, L_{1}, \hat{L}_{0}, \hat{L}_{1} \in[0, \infty)$ be the real numbers given by $L_{0}=|F|_{\operatorname{Lip}\left(H, H_{-\alpha}\right)}, L_{1}=|B|_{\operatorname{Lip}\left(H, H S\left(U, H_{-}\right)\right)}, \hat{L}_{0}=\|F(0)\|_{H_{\alpha}}$, and $\hat{L}_{1}=\|B(0)\|_{H S\left(U, H_{\beta}\right)}$. We note that for all $t \in(0, T], X, Y \in \mathcal{L}^{p}(\mathbb{P} ; H)$ it holds that

$$
\begin{array}{cl}
\|F(X)-F(Y)\|_{L^{p}\left(\mathbb{P} ; H_{-\alpha}\right)} & \leq L_{0}\|X-Y\|_{L^{p}(\mathbb{P} ; H)}, \quad\|F(0)\|_{L^{p}\left(\mathbb{P} ; H_{-\alpha}\right)} \leq \hat{L}_{0}, \\
\|B(X)-B(Y)\|_{L^{p}\left(\mathbb{P} ; H S\left(U, H_{-\beta}\right)\right)} \leq L_{1}\|X-Y\|_{L^{p}(\mathbb{P} ; H)}, \quad\|B(0)\|_{L^{p}\left(\mathbb{P} ; H S\left(U, H_{-\beta}\right)\right)} \leq \hat{L}_{1} .
\end{array}
$$

We can hence apply Corollary 2.8 and Theorem 2.9. More specifically, an application of Theorem 2.9 (with $\delta=\delta, \lambda=\delta, \hat{\alpha}=\hat{\beta}=0, L_{0}=|F|_{\operatorname{Lip}\left(H, H_{-\alpha}\right)}, \quad \hat{L}_{0}=\|F(0)\|_{H_{-\alpha}}$, $L_{1}=|B|_{\operatorname{Lip}\left(H, H S\left(U, H_{-\beta}\right)\right)}$, and $\hat{L}_{1}=\|B(0)\|_{H S\left(U, H_{-\beta}\right)}$ for $\delta \in[0, \hat{\delta})$ in the notation of Theorem 2.9]) proves (iㅣ), proves that for all $p \in[2, \infty), \delta \in[0, \hat{\delta}), x \in H_{-\delta}$ it holds that

$$
\begin{aligned}
& \sup _{t \in(0, T]}\left[t^{\delta}\left\|X_{t}^{x}\right\|_{L^{p}(\mathbb{P} ; H)}\right] \leq \Theta_{A, \eta, p, T}^{\alpha, \beta, \delta}\left(|F|_{\operatorname{Lip}\left(H, H_{-\alpha}\right)},|B|_{\operatorname{Lip}\left(H, H S\left(U, H_{-\beta}\right)\right)}\right) \\
& \cdot\left[\chi_{A, \eta}^{\delta, T}\|x\|_{H_{-\delta}}+\chi_{A, \eta}^{\alpha, T}\|F(0)\|_{H_{-\alpha}} T^{(\delta+1-\alpha)} \mathbb{B}(1-\alpha, 1)\right. \\
& \left.\quad+\frac{\chi_{A, \eta}^{\beta, T}\|B(0)\|_{H S\left(U, H_{-\beta}\right)} T^{(\delta+1 / 2-\beta)}|p(p-1) \mathbb{B}(1-2 \beta, 1)|^{1 / 2}}{\sqrt{2}}\right]<\infty
\end{aligned}
$$

and proves that for all $p \in[2, \infty), \delta \in[0, \hat{\delta}), x \in H_{-\delta}, \varrho \in[0, \min \{1-\alpha, 1 / 2-\beta\}), s, t \in(0, T]$ with $s<t$ it holds that

$$
\begin{aligned}
& \left\|X_{s}^{x}-X_{t}^{x}\right\|_{L^{p}(\mathbb{P} ; H)} \leq|s-t|^{\varrho} \\
& \cdot\left[\frac{\kappa_{A, \eta}^{\varrho, T} \chi_{A, \eta}^{\varrho+\delta, T}\|x\|_{H_{-\delta}}}{s^{(\varrho+\delta)}}+|T \vee 1|^{\delta}\left[\frac{\chi_{A, \eta}^{\alpha, T}|s-t|^{(1-\alpha-\varrho)}}{(1-\alpha) s^{\delta}}+\frac{\kappa_{A, \eta}^{\varrho, T} \chi_{A, \eta}^{\varrho+\alpha, T} \mathbb{B}(1-\alpha-\varrho, 1-\delta)}{s^{(\varrho+\alpha+\delta-1)}}\right]\right. \\
& \cdot\left(\|F(0)\|_{H_{-\alpha}}+|F|_{\operatorname{Lip}\left(H_{, H} H_{-\alpha}\right)} \sup _{u \in(0, T]} u^{\delta}\left\|X_{u}^{x}\right\|_{L^{p}(\mathbb{P} ; H)}\right)+|T \vee 1|^{\left.\delta \mathbb{1}_{(0, \infty)}\left(|B|_{\operatorname{Lip}(H, H S(U, H-\beta}\right)\right)} \\
& \cdot \sqrt{\frac{p(p-1)}{2}}\left(\|B(0)\|_{H S\left(U, H_{-\beta}\right)}+|B|_{\operatorname{Lip}\left(H, H S\left(U, H_{-\beta}\right)\right)} \sup _{u \in(0, T]} u^{\delta}\left\|X_{u}^{x}\right\|_{L^{p}(\mathbb{P} ; H)}\right)
\end{aligned}
$$

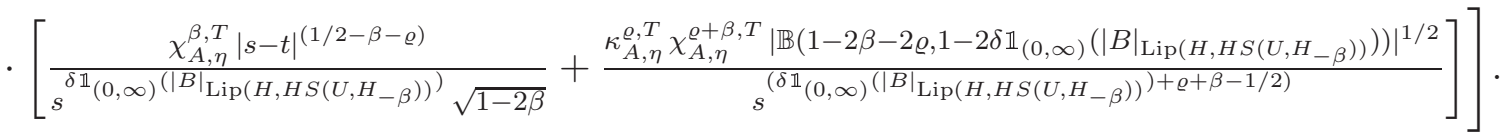


Observe that (84) establishes (iii) and note that (iii) and (85) establish (iv). In addition, an application of Corollary 2.8 (with $X^{1}=X^{x}, X^{2}=X^{y}, \delta=\delta$, and $\lambda=\delta$ for $x, y \in H_{-\delta}$, $\delta \in[0, \hat{\delta})$ in the notation of Corollary 2.8) ensures that for all $p \in[2, \infty), \delta \in[0, \hat{\delta}), x, y \in H_{-\delta}$ it holds that

$$
\begin{aligned}
& \sup _{t \in(0, T]}\left[t^{\delta}\left\|X_{t}^{x}-X_{t}^{y}\right\|_{L^{p}(\mathbb{P} ; H)}\right] \\
& \leq \chi_{A, \eta}^{\delta, T}\|x-y\|_{H_{-\delta}} \Theta_{A, \eta, p, T}^{\alpha, \beta, \delta}\left(|F|_{\operatorname{Lip}\left(H, H_{-\alpha}\right)},|B|_{\operatorname{Lip}\left(H, H S\left(U, H_{-\beta}\right)\right)}\right)<\infty .
\end{aligned}
$$

This establishes (iii). The proof of Corollary 2.10 is thus completed.

\section{Examples and counterexamples for SEEs with irregu- lar initial values}

Corollary 2.10 in Subsection 2.4 above establishes existence, uniqueness, and regularity properties for solutions of parabolic SEEs. In this section we first illustrate the statement of Corollary 2.10 in the case of semilinear stochastic heat equations with space-time white noise and periodic boundary conditions; see Corollary 3.1 in Subsection 3.2 below. Roughly speaking, Corollary 3.1 shows existence and uniqueness of solutions of the considered stochastic heat equation provided that the initial value lies in $\cup_{\delta \in(-1 / 2, \infty)} H_{\delta}$ where $H_{r}, r \in \mathbb{R}$, is a family of interpolation spaces associated to the Laplacian with periodic boundary conditions. Corollary 3.1 applies, in particular, to the continuous version of the parabolic Anderson model. Thereafter, we illustrate in Proposition 3.2 in Subsection 3.2, in Proposition 3.4 in Subsection 3.3, and in Proposition 3.5 in Subsection 3.4 by means of several example SEEs that the statement of Corollary 2.10 can in general not be improved. Moreover, we illustrate in Proposition 3.3 in Subsection 3.2 in the case of a specific linear example SEE with regular noise that the statement of Corollary 2.10 can be improved.

\subsection{Setting}

Throughout this section the following setting is sometimes used. Let $\left(H,\|\cdot\|_{H},\langle\cdot, \cdot\rangle_{H}\right)$ be a separable $\mathbb{R}$-Hilbert space, let $T \in(0, \infty), \eta, \delta \in \mathbb{R}$, let $\left(\Omega, \mathcal{F}, \mathbb{P},\left(\mathcal{F}_{t}\right)_{t \in[0, T]}\right)$ be a stochastic basis, let $A: D(A) \subseteq H \rightarrow H$ be a diagonal linear operator with spectrum $(A) \subseteq(-\infty, \eta)$, and let $\left(H_{r},\|\cdot\|_{H_{r}},\langle\cdot, \cdot\rangle_{H_{r}}\right), r \in \mathbb{R}$, be a family of interpolation spaces associated to $\eta-A$.

\subsection{Stochastic heat equations with linear multiplicative noise}

Corollary 3.1. Let $\left(H,\|\cdot\|_{H},\langle\cdot, \cdot\rangle_{H}\right)=\left(L^{2}\left(\mu_{(0,1)} ; \mathbb{R}\right),\|\cdot\|_{L^{2}\left(\mu_{(0,1)} ; \mathbb{R}\right)},\langle\cdot, \cdot\rangle_{L^{2}\left(\mu_{(0,1)} ; \mathbb{R}\right)}\right)$, let $T, \eta \in$ $(0, \infty), \beta \in(1 / 4,1 / 2), f, b \in \operatorname{Lip}(\mathbb{R}, \mathbb{R})$, let $\left(\Omega, \mathcal{F}, \mathbb{P},\left(\mathcal{F}_{t}\right)_{t \in[0, T]}\right)$ be a stochastic basis, let $\left(W_{t}\right)_{t \in[0, T]}$ be an $\operatorname{Id}_{H}$-cylindrical $\left(\mathcal{F}_{t}\right)_{t \in[0, T]}$-Wiener process, let $A: D(A) \subseteq H \rightarrow H$ be the Laplacian with periodic boundary conditions on $H$, let $\left(H_{r},\|\cdot\|_{H_{r}},\langle\cdot, \cdot\rangle_{H_{r}}\right), r \in \mathbb{R}$, be a family of interpolation spaces associated to $\eta-A$, and let $F: H \rightarrow H$ and $B: H \rightarrow H S\left(H, H_{-\beta}\right)$ satisfy for 
all $v \in \mathcal{L}^{2}\left(\mu_{(0,1)} ; \mathbb{R}\right), u \in \mathcal{C}([0,1], \mathbb{R})$ that $F\left([v]_{\mu_{(0,1)}, \mathcal{B}(\mathbb{R})}\right)=\left[\{f(v(x))\}_{x \in(0,1)}\right]_{\mu_{(0,1)}, \mathcal{B}(\mathbb{R})}$ and $B\left([v]_{\mu_{(0,1)}, \mathcal{B}(\mathbb{R})}\right)\left[\left.u\right|_{(0,1)}\right]_{\mu_{(0,1)}, \mathcal{B}(\mathbb{R})}=\left[\{b(v(x)) \cdot u(x)\}_{x \in(0,1)}\right]_{\mu_{(0,1)}, \mathcal{B}(\mathbb{R})}$. Then

(i) there exist up-to-modifications unique $\left(\mathcal{F}_{t}\right)_{t \in[0, T]}$-predictable stochastic processes $X^{x}:[0, T] \times$ $\Omega \rightarrow H_{-\delta}, x \in H_{-\delta}, \delta \in[0,1 / 2)$, which fulfill for all $p \in[2, \infty), \delta \in[0,1 / 2), x \in H_{-\delta}$, $t \in[0, T]$ that $X^{x}((0, T] \times \Omega) \subseteq H$, that $\sup _{s \in(0, T]} s^{\delta}\left\|X_{s}^{x}\right\|_{L^{p}(\mathbb{P} ; H)}<\infty$, and $\mathbb{P}$-a.s. that

$$
X_{t}^{x}=e^{t A} x+\int_{0}^{t} e^{(t-s) A} F\left(X_{s}^{x}\right) \mathrm{d} s+\int_{0}^{t} e^{(t-s) A} B\left(X_{s}^{x}\right) \mathrm{d} W_{s}
$$

(ii) and for all $p \in[2, \infty), \delta \in[0,1 / 2)$ it holds that

$$
\sup _{\substack{x, y \in H_{-\delta}, t \in(0, T] \\ x \neq y}} \sup _{\substack{\delta \neq y \\ \text { max }\left\{1,\|x\|_{H_{-\delta}}\right\}}}\left[\frac{t^{\delta}\left\|X_{t}^{x}\right\|_{L^{p}(\mathbb{P} ; H)}\left\|X_{t}^{x}-X_{t}^{y}\right\|_{L^{p}(\mathbb{P} ; H)}}{\|x-y\|_{H_{-\delta}}}\right]<\infty .
$$

Proposition 3.2. Let $\left(H,\|\cdot\|_{H},\langle\cdot, \cdot\rangle_{H}\right)=\left(L^{2}\left(\mu_{(0,1)} ; \mathbb{R}\right),\|\cdot\|_{L^{2}\left(\mu_{(0,1)} ; \mathbb{R}\right)},\langle\cdot, \cdot\rangle_{L^{2}\left(\mu_{(0,1)} ; \mathbb{R}\right)}\right)$, let $T, \eta$, $\nu \in(0, \infty), r \in[0, \infty), \delta \in \mathbb{R}, \beta \in\left(\frac{1}{4}, \frac{1}{2}\right)$, let $\left(\Omega, \mathcal{F}, \mathbb{P},\left(\mathcal{F}_{t}\right)_{t \in[0, T]}\right)$ be a stochastic basis, let $\left(W_{t}\right)_{t \in[0, T]}$ be an $\operatorname{Id}_{H}$-cylindrical $\left(\mathcal{F}_{t}\right)_{t \in[0, T]}$-Wiener process, let $\left(b_{n}\right)_{n \in \mathbb{Z}} \subseteq H$ satisfy for all $n \in \mathbb{N}$ that $b_{0}=\left[\{1\}_{x \in(0,1)}\right]_{\mu_{(0,1)}, \mathcal{B}(\mathbb{R})}, b_{n}=\left[\{\sqrt{2} \cos (2 n \pi x)\}_{x \in(0,1)}\right]_{\mu_{(0,1)}, \mathcal{B}(\mathbb{R})}$, and $b_{-n}=$ $\left[\{\sqrt{2} \sin (2 n \pi x)\}_{x \in(0,1)}\right]_{\mu_{(0,1)}, \mathcal{B}(\mathbb{R})}$, let $A: D(A) \subseteq H \rightarrow H$ be the linear operator which satisfies $D(A)=\left\{v \in H: \sum_{n \in \mathbb{Z}}\left|n^{2}\left\langle b_{n}, v\right\rangle_{H}\right|^{2}<\infty\right\}$ and which satisfies for all $v \in D(A)$ that Av $=$ $-\nu \sum_{n \in \mathbb{Z}} n^{2}\left\langle b_{n}, v\right\rangle_{H} b_{n}$, let $\left(H_{q},\|\cdot\|_{H_{q}},\langle\cdot, \cdot\rangle_{H_{q}}\right), q \in \mathbb{R}$, be a family of interpolation spaces associated to $\eta-A$, let $\xi \in \mathcal{M}\left(\mathcal{F}_{0}, \mathcal{B}\left(H_{\delta}\right)\right), B \in L\left(H, H S\left(H, H_{-\beta}\right)\right)$ satisfy for all $v \in \mathcal{L}^{2}\left(\mu_{(0,1)} ; \mathbb{R}\right)$, $u \in \mathcal{C}([0,1], \mathbb{R})$ that $B\left([v]_{\mu_{(0,1)}, \mathcal{B}(\mathbb{R})}\right)\left[\left.u\right|_{(0,1)}\right]_{\mu_{(0,1)}, \mathcal{B}(\mathbb{R})}=\left[\{v(x) \cdot u(x)\}_{x \in(0,1)}\right]_{\mu_{(0,1)}, \mathcal{B}(\mathbb{R})}$, and let $X:[0, T] \times \Omega \rightarrow H_{\delta}$ be an $\left(\mathcal{F}_{t}\right)_{t \in[0, T]}$-predictable stochastic process which satisfies for all $t \in(0, T]$ that $X((0, T] \times \Omega) \subseteq H$, that

$$
\mathbb{E}\left[\left\|e^{t A} \xi\right\|_{H_{-r}}^{2}\right]+\int_{0}^{t} \mathbb{E}\left[\left\|e^{(t-s) A} B\left(X_{s}\right)\right\|_{H S\left(H, H_{-r}\right)}^{2}\right] d s<\infty,
$$

and $\mathbb{P}$-a.s. that $X_{t}=e^{t A} \xi+\int_{0}^{t} e^{(t-s) A} B\left(X_{s}\right) d W_{s}$. Then

(i) it holds that $\mathbb{P}\left(\xi \in H_{-1 / 2}\right)=1$ and

(ii) it holds for all $t \in(0, T]$ that

$$
2^{-1 / 2} \eta^{-r}\left(1-e^{-2 \eta t}\right)^{1 / 2}\|\xi\|_{L^{2}\left(\mathbb{P} ; H_{-1 / 2}\right)} \leq\left\|X_{t}\right\|_{L^{2}\left(\mathbb{P} ; H_{-r}\right)}<\infty .
$$

Proof. Throughout this proof let $\kappa_{k} \in[0, \infty], k \in \mathbb{Z}$, be the extended real numbers which satisfy for all $k \in \mathbb{Z}$ that

$$
\kappa_{k}=\sup _{x \in\left(\cap_{s \in \mathbb{R}} H_{s}\right) \backslash\{0\}} \frac{\left\|B(x) b_{k}\right\|_{H_{-r-1}}^{2}}{\|x\|_{H_{-r}}^{2}} .
$$


Observe that the product rule for differentiation and the fact that the mapping $\mathcal{C}([0,1], \mathbb{R}) \ni$ $v \mapsto\left[\left.v\right|_{(0,1)}\right]_{\mu_{(0,1)}, \mathcal{B}(\mathbb{R})} \in H_{1 / 2}$ is continuous ensures that for all $n \in \mathbb{N}$ it holds that $\forall u, v \in$ $\cap_{s \in \mathbb{R}} H_{s}: u \cdot v \in \cap_{s \in \mathbb{R}} H_{s}$ and $\sup _{u, v \in\left(\cap_{s \in \mathbb{R}} H_{s}\right) \backslash\{0\}} \frac{\|u \cdot v\|_{H_{n}}}{\|u\|_{H_{n}}\|v\|_{H_{n}}}<\infty$. This implies that for all $k \in \mathbb{Z}, n \in \mathbb{N}_{0}$ it holds that

$$
\begin{aligned}
& \quad \sup _{x \in\left(\cap_{s \in \mathbb{R}} H_{s}\right) \backslash\{0\}} \frac{\left\|B(x) b_{k}\right\|_{H_{-n}}}{\|x\|_{H_{-n}}}=\sup _{x, u \in\left(\cap_{s \in \mathbb{R}} H_{s}\right) \backslash\{0\}} \frac{\left|\left\langle u, B(x) b_{k}\right\rangle_{H}\right|}{\|x\|_{H_{-n}}\|u\|_{H_{n}}} \\
& =\sup _{x, u \in\left(\cap_{s \in \mathbb{R}} H_{s}\right) \backslash\{0\}} \frac{\left|\left\langle u \cdot b_{k}, x\right\rangle_{H}\right|}{\|x\|_{H_{-n}}\|u\|_{H_{n}}}=\sup _{x, u \in\left(\cap_{s \in \mathbb{R}} H_{s}\right) \backslash\{0\}} \frac{\left|\left\langle(\eta-A)^{n}\left(u \cdot b_{k}\right),(\eta-A)^{-n} x\right\rangle_{H}\right|}{\|x\|_{H_{-n}}\|u\|_{H_{n}}} \\
& \leq \sup _{u \in\left(\cap_{s \in \mathbb{R}} H_{s}\right) \backslash\{0\}} \frac{\left\|u \cdot b_{k}\right\|_{H_{n}}}{\|u\|_{H_{n}}}<\infty .
\end{aligned}
$$

Hence, we obtain that for all $k \in \mathbb{Z}$ it holds that

$$
\begin{aligned}
& \kappa_{k}=\sup _{x \in\left(\cap_{s \in \mathbb{R}} H_{s}\right) \backslash\{0\}} \frac{\left\|B(x) b_{k}\right\|_{H_{-r-1}}^{2}}{\|x\|_{H_{-r}}^{2}} \\
& \leq\left\|(\eta-A)^{-r-1-\lceil-r-1\rceil_{1}}\right\|_{L(H)}^{2}\left[\sup _{x \in\left(\cap_{s \in \mathbb{R}} H_{s}\right) \backslash\{0\}} \frac{\left\|B(x) b_{k}\right\|_{H_{\lceil-r-1\rceil_{1}}}}{\|x\|_{H_{-r}}}\right]^{2} \\
& \leq\left\|(\eta-A)^{-r-1-\lceil-r-1\rceil_{1}}\right\|_{L(H)}^{2}\left[\sup _{x \in\left(\cap_{s \in \mathbb{R}} H_{s}\right) \backslash\{0\}} \frac{\|x\|_{H_{\lceil-r-1\rceil_{1}}}}{\|x\|_{H_{-r}}}\right]^{2} \\
& \cdot\left[\sup _{x \in\left(\cap_{s \in \mathbb{R}} H_{s}\right) \backslash\{0\}} \frac{\left\|B(x) b_{k}\right\|_{H_{\lceil-r-1\rceil_{1}}}}{\|x\|_{H_{\lceil-r-1\rceil_{1}}}}\right]^{2} \\
& =\left\|(\eta-A)^{-r-1-\lceil-r-1\rceil_{1}}\right\|_{L(H)}^{2}\left\|(\eta-A)^{r+\lceil-r-1\rceil_{1}}\right\|_{L(H)}^{2} \\
& \cdot\left[\sup _{x \in\left(\cap_{s \in \mathbb{R}} H_{s}\right) \backslash\{0\}} \frac{\left\|B(x) b_{k}\right\|_{H_{\lceil-r-1\rceil_{1}}}}{\|x\|_{H_{\lceil-r-1\rceil_{1}}}}\right]^{2}<\infty .
\end{aligned}
$$

In the next step we observe that for all $t \in(0, T]$ it holds $\mathbb{P}$-a.s. that

$$
\begin{aligned}
& \left\|X_{t}\right\|_{H_{-r}}^{2}=\left\|e^{t A} \xi+\int_{0}^{t} e^{(t-s) A} B\left(X_{s}\right) d W_{s}\right\|_{H_{-r}}^{2} \\
& =\left\|e^{t A} \xi\right\|_{H_{-r}}^{2}+2\left\langle e^{t A} \xi, \int_{0}^{t} e^{(t-s) A} B\left(X_{s}\right) d W_{s}\right\rangle_{H_{-r}}+\left\|\int_{0}^{t} e^{(t-s) A} B\left(X_{s}\right) d W_{s}\right\|_{H_{-r}}^{2} .
\end{aligned}
$$

Combining (94) with Itô's isometry and the assumption that $\forall t \in(0, T]: \mathbb{E}\left[\left\|e^{t A} \xi\right\|_{H_{-r}}^{2}\right]+$ 
$\int_{0}^{t} \mathbb{E}\left[\left\|e^{(t-s) A} B\left(X_{s}\right)\right\|_{H S\left(H, H_{-r}\right)}^{2}\right] d s<\infty$ proves that for all $t \in(0, T]$ it holds that

$$
\begin{aligned}
\mathbb{E} & {\left[\left\|X_{t}\right\|_{H_{-r}}^{2}\right]=\mathbb{E}\left[\left\|e^{t A} \xi\right\|_{H_{-r}}^{2}\right]+2 \mathbb{E}\left[\left\langle e^{t A} \xi, \int_{0}^{t} e^{(t-s) A} B\left(X_{s}\right) d W_{s}\right\rangle_{H_{-r}}\right] } \\
& +\mathbb{E}\left[\left\|\int_{0}^{t} e^{(t-s) A} B\left(X_{s}\right) d W_{s}\right\|_{H_{-r}}^{2}\right] \\
= & \mathbb{E}\left[\left\|e^{t A} \xi\right\|_{H_{-r}}^{2}\right]+2 \mathbb{E}\left[\left\langle e^{t A} \xi, \mathbb{E}\left[\int_{0}^{t} e^{(t-s) A} B\left(X_{s}\right) d W_{s} \mid \mathcal{F}_{0}\right]\right\rangle_{H_{-r}}\right] \\
& +\int_{0}^{t} \mathbb{E}\left[\left\|e^{(t-s) A} B\left(X_{s}\right)\right\|_{H S\left(H, H_{-r}\right)}^{2}\right] d s \\
= & \mathbb{E}\left[\left\|e^{t A} \xi\right\|_{H_{-r}}^{2}\right]+\int_{0}^{t} \mathbb{E}\left[\left\|e^{(t-s) A} B\left(X_{s}\right)\right\|_{H S\left(H, H_{-r}\right)}^{2}\right] d s \\
= & \mathbb{E}\left[\left\|e^{t A} \xi\right\|_{H_{-r}}^{2}\right]+\sum_{k \in \mathbb{Z}} \int_{0}^{t} \mathbb{E}\left[\left\|e^{(t-s) A} B\left(X_{s}\right) b_{k}\right\|_{H_{-r}}^{2}\right] d s<\infty .
\end{aligned}
$$

Moreover, we note that for all $k \in \mathbb{Z}, t \in(0, T], s \in(0, t)$ it holds $\mathbb{P}$-a.s. that

$$
\begin{aligned}
& \left\|e^{(t-s) A} B\left(X_{s}\right) b_{k}\right\|_{H_{-r}}^{2}=\left\|e^{(t-s) A} B\left(e^{s A} \xi+\int_{0}^{s} e^{(s-u) A} B\left(X_{u}\right) d W_{u}\right) b_{k}\right\|_{H_{-r}}^{2} \\
= & \left\|e^{(t-s) A} B\left(e^{s A} \xi\right) b_{k}\right\|_{H_{-r}}^{2}+\left\|e^{(t-s) A} B\left(\int_{0}^{s} e^{(s-u) A} B\left(X_{u}\right) d W_{u}\right) b_{k}\right\|_{H_{-r}}^{2} \\
& +2\left\langle e^{(t-s) A} B\left(e^{s A} \xi\right) b_{k}, e^{(t-s) A} B\left(\int_{0}^{s} e^{(s-u) A} B\left(X_{u}\right) d W_{u}\right) b_{k}\right\rangle_{H_{-r}} \\
\geq & \left\|e^{(t-s) A} B\left(e^{s A} \xi\right) b_{k}\right\|_{H_{-r}}^{2} \\
& +2\left\langle e^{(t-s) A} B\left(e^{s A} \xi\right) b_{k}, \int_{0}^{s} e^{(t-s) A} B\left(e^{(s-u) A} B\left(X_{u}\right) d W_{u}\right) b_{k}\right\rangle_{H_{-r}} .
\end{aligned}
$$

This and assumption (89) imply that for all $k \in \mathbb{Z}, t \in(0, T], s \in(0, t)$ it holds that

$$
\begin{aligned}
& \mathbb{E}\left[\sum_{n \in \mathbb{Z}} \int_{0}^{s}\left\|e^{(t-s) A} B\left(e^{(s-u) A} B\left(X_{u}\right) b_{n}\right) b_{k}\right\|_{H_{-r}}^{2} d u\right] \\
& \leq\left\|e^{(t-s) A}\right\|_{L\left(H_{-r-1}, H_{-r}\right)}^{2} \mathbb{E}\left[\sum_{n \in \mathbb{Z}} \int_{0}^{s}\left\|B\left(e^{(s-u) A} B\left(X_{u}\right) b_{n}\right) b_{k}\right\|_{H_{-r-1}}^{2} d u\right] \\
& \leq \kappa_{k}\left\|e^{(t-s) A}\right\|_{L\left(H_{-r-1}, H_{-r}\right)}^{2} \mathbb{E}\left[\sum_{n \in \mathbb{Z}} \int_{0}^{s}\left\|e^{(s-u) A} B\left(X_{u}\right) b_{n}\right\|_{H_{-r}}^{2} d u\right] \\
& =\kappa_{k}\left\|e^{(t-s) A}\right\|_{L\left(H_{-r-1}, H_{-r}\right)}^{2} \mathbb{E}\left[\int_{0}^{s}\left\|e^{(s-u) A} B\left(X_{u}\right)\right\|_{H S\left(H, H_{-r}\right)}^{2} d u\right]<\infty
\end{aligned}
$$

and

$$
\mathbb{E}\left[\left\|e^{(t-s) A} B\left(e^{s A} \xi\right) b_{k}\right\|_{H_{-r}}^{2}\right] \leq \kappa_{k}\left\|e^{(t-s) A}\right\|_{L\left(H_{-r-1}, H_{-r}\right)}^{2} \mathbb{E}\left[\left\|e^{s A} \xi\right\|_{H_{-r}}^{2}\right]<\infty
$$


Combining (96) with (97)-(98) proves that for all $k \in \mathbb{Z}, t \in(0, T], s \in(0, t)$ it holds that

$$
\begin{aligned}
\mathbb{E} & {\left[\left\|e^{(t-s) A} B\left(X_{s}\right) b_{k}\right\|_{H_{-r}}^{2}\right] \geq \mathbb{E}\left[\left\|e^{(t-s) A} B\left(e^{s A} \xi\right) b_{k}\right\|_{H_{-r}}^{2}\right] } \\
& +2 \mathbb{E}\left[\left\langle e^{(t-s) A} B\left(e^{s A} \xi\right) b_{k}, \int_{0}^{s} e^{(t-s) A} B\left(e^{(s-u) A} B\left(X_{u}\right) d W_{u}\right) b_{k}\right\rangle_{H_{-r}}\right] \\
= & \mathbb{E}\left[\left\|e^{(t-s) A} B\left(e^{s A} \xi\right) b_{k}\right\|_{H_{-r}}^{2}\right] \\
& +2 \mathbb{E}\left[\left\langle e^{(t-s) A} B\left(e^{s A} \xi\right) b_{k}, \mathbb{E}\left[\int_{0}^{s} e^{(t-s) A} B\left(e^{(s-u) A} B\left(X_{u}\right) d W_{u}\right) b_{k} \mid \mathcal{F}_{0}\right]\right\rangle_{H_{-r}}\right] \\
= & \mathbb{E}\left[\left\|e^{(t-s) A} B\left(e^{s A} \xi\right) b_{k}\right\|_{H_{-r}}^{2}\right] .
\end{aligned}
$$

Combining this with (95) ensures that for all $t \in(0, T]$ it holds that

$$
\begin{aligned}
&>\mathbb{E}\left[\left\|X_{t}\right\|_{H_{-r}}^{2}\right] \geq \mathbb{E}\left[\left\|e^{t A} \xi\right\|_{H_{-r}}^{2}\right]+\sum_{k \in \mathbb{Z}} \int_{0}^{t} \mathbb{E}\left[\left\|e^{(t-s) A} B\left(e^{s A} \xi\right) b_{k}\right\|_{H_{-r}}^{2}\right] d s \\
& \geq \int_{0}^{t} \mathbb{E}\left[\left\|e^{(t-s) A} B\left(e^{s A} \xi\right)\right\|_{H S\left(H, H_{-r}\right)}^{2}\right] d s=\int_{0}^{t} \mathbb{E}\left[\left\|(\eta-A)^{-r} e^{(t-s) A} B\left(e^{s A} \xi\right)\right\|_{H S(H)}^{2}\right] d s \\
&=\int_{0}^{t} \mathbb{E}\left[\left\|B\left(e^{s A} \xi\right) e^{(t-s) A}(\eta-A)^{-r}\right\|_{H S(H)}^{2}\right] d s \\
&=\sum_{n \in \mathbb{Z}} \int_{0}^{t} \mathbb{E}\left[\left\|B\left(e^{s A} \xi\right) e^{(t-s) A}(\eta-A)^{-r} b_{n}\right\|_{H}^{2}\right] d s \\
& \geq \int_{0}^{t} \mathbb{E}\left[\left\|B\left(e^{s A} \xi\right) e^{(t-s) A}(\eta-A)^{-r} b_{0}\right\|_{H}^{2}\right] d s \\
&= \eta^{-2 r} \int_{0}^{t} \mathbb{E}\left[\left\|B\left(e^{s A} \xi\right) b_{0}\right\|_{H}^{2}\right] d s=\eta^{-2 r} \int_{0}^{t} \mathbb{E}\left[\left\|e^{s A} \xi\right\|_{H}^{2}\right] d s \\
&= \eta^{-2 r} \sum_{n \in \mathbb{Z}} \int_{0}^{t} \mathbb{E}\left[\left|\left\langle e^{s A} b_{n}, \xi\right\rangle_{H}\right|^{2}\right] d s=\eta^{-2 r} \sum_{n \in \mathbb{Z}} \int_{0}^{t} e^{-2\left(\nu n^{2}+\eta\right) s} e^{2 \eta s} \mathbb{E}\left[\left|\left\langle b_{n}, \xi\right\rangle_{H}\right|^{2}\right] d s \\
& \geq \eta^{-2 r} \sum_{n \in \mathbb{Z}} \int_{0}^{t} e^{-2\left(\nu n^{2}+\eta\right) s} \mathbb{E}\left[\left|\left\langle b_{n}, \xi\right\rangle_{H}\right|^{2}\right] d s=\eta^{-2 r} \sum_{n \in \mathbb{Z}} \frac{\left(1-e^{-2\left(\nu n^{2}+\eta\right) t}\right) \mathbb{E}\left[\left|\left\langle b_{n}, \xi\right\rangle_{H}\right|^{2}\right]}{2\left(\nu n^{2}+\eta\right)} \\
&=\frac{1}{2 \eta^{2 r}} \sum_{n \in \mathbb{Z}}\left(1-e^{-2\left(\nu n^{2}+\eta\right) t}\right) \mathbb{E}\left[\left|\left\langle(\eta-A)^{-1 / 2} b_{n}, \xi\right\rangle_{H}\right|^{2}\right] \\
& \geq \frac{\left(1-e^{-2 \eta t}\right)}{2 \eta^{2 r}} \sum_{n \in \mathbb{Z}} \mathbb{E}\left[\left|\left\langle(\eta-A)^{-1 / 2} b_{n}, \xi\right\rangle_{H}\right|^{2}\right] .
\end{aligned}
$$

In particular, we obtain that $\mathbb{E}\left[\sum_{n \in \mathbb{Z}}\left|\left\langle(\eta-A)^{-1 / 2} b_{n}, \xi\right\rangle_{H}\right|^{2}\right]<\infty$. Therefore, it holds that $\mathbb{P}\left(\xi \in H_{-1 / 2}\right)=1$. This and (100) complete the proof of Proposition 3.2 . 
Proposition 3.3 (Positive example). Assume the setting in Subsection [3.1, let $k \in \mathbb{N}, \xi \in$ $\mathcal{M}\left(\mathcal{F}_{0}, \mathcal{B}\left(H_{\delta}\right)\right),\left(L_{i}\right)_{i \in\{1,2, \ldots, k\}} \subseteq L(H), B \in L\left(H, H S\left(\mathbb{R}^{k}, H\right)\right)$ satisfy for all $i, j \in\{1,2, \ldots, k\}$, $v \in H, u \in D(A), \mathbf{y}=\left(y_{1}, y_{2}, \ldots, y_{k}\right) \in \mathbb{R}^{k}$ that $L_{i}(D(A)) \subseteq D(A), L_{i} L_{j} u-L_{j} L_{i} u=$ $L_{i} A u-A L_{i} u=0$, and $B(v) \mathbf{y}=\sum_{l=1}^{k} y_{l} L_{l} v$, let $W=\left(W^{(1)}, W^{(2)}, \ldots, W^{(k)}\right):[0, T] \times \Omega \rightarrow \mathbb{R}^{k}$ be a $k$-dimensional standard $\left(\mathcal{F}_{t}\right)_{t \in[0, T]}$-Brownian motion with continuous sample paths, and let $X:[0, T] \times \Omega \rightarrow H_{\delta}$ satisfy for all $t \in[0, T]$ that

$$
X_{t}=\exp \left(t A+\sum_{i=1}^{k}\left[W_{t}^{(i)} L_{i}-\frac{1}{2} t\left(L_{i}\right)^{2}\right]\right) \xi
$$

Then $X$ has continuous sample paths and for all $r \in \mathbb{R}, t \in[0, T]$ it holds $\mathbb{P}$-a.s. that $\int_{0}^{t}\left\|e^{(t-s) A} B\left(X_{s}\right)\right\|_{H S\left(\mathbb{R}^{k}, H_{r}\right)}^{2} d s<\infty$ and $X_{t}=e^{t A} \xi+\int_{0}^{t} e^{(t-s) A} B\left(X_{s}\right) d W_{s}$.

Proof. Throughout this proof let $r \in[0, \infty)$ and let $\varphi \in \mathcal{C}\left([0, T] \times \mathbb{R}^{k} \times H_{r}, H_{r}\right)$ be the mapping with the property that for all $t \in[0, T], \mathbf{y}=\left(y_{1}, y_{2}, \ldots, y_{k}\right) \in \mathbb{R}^{k}, v \in H_{r}$ it holds that $\varphi(t, \mathbf{y}, v)=\exp \left(\sum_{i=1}^{k}\left[y_{i} L_{i}-\frac{1}{2} t\left(L_{i}\right)^{2}\right]\right) v$. Note that the assumption that $W$ has continuous sample paths ensures that $X$ also has continuous sample paths. Next observe that $\varphi \in \mathcal{C}^{2}\left([0, T] \times \mathbb{R}^{k} \times H_{r}, H_{r}\right)$. Itô's formula (cf., e.g., Theorem 2.4 in Brzeźniak, Van Neerven, Veraar \& Weis [5]) therefore implies that for all $t \in(0, T]$ it holds $\mathbb{P}$-a.s. that

$$
\begin{aligned}
& \int_{0}^{t}\left\|\left(\frac{\partial}{\partial \mathbf{y}} \varphi\right)\left(s, W_{s}, e^{t A} \xi\right)\right\|_{H S\left(\mathbb{R}^{k}, H_{r}\right)}^{2} \mathrm{~d} s=\int_{0}^{t} \sum_{i=1}^{k}\left\|\left(\frac{\partial}{\partial y_{i}} \varphi\right)\left(s, W_{s}, e^{t A} \xi\right)\right\|_{H_{r}}^{2} \mathrm{~d} s \\
& =\int_{0}^{t} \sum_{i=1}^{k}\left\|e^{(t-s) A}\left(\frac{\partial}{\partial y_{i}} \varphi\right)\left(s, W_{s}, e^{s A} \xi\right)\right\|_{H_{r}}^{2} \mathrm{~d} s=\int_{0}^{t}\left\|e^{(t-s) A} B\left(X_{s}\right)\right\|_{H S\left(\mathbb{R}^{k}, H_{r}\right)}^{2} \mathrm{~d} s<\infty
\end{aligned}
$$

and

$$
\begin{aligned}
X_{t}= & \varphi\left(t, W_{t}, e^{t A} \xi\right)=\varphi\left(0,0, e^{t A} \xi\right)+\int_{0}^{t}\left(\frac{\partial}{\partial s} \varphi\right)\left(u, W_{u}, e^{t A} \xi\right) \mathrm{d} u \\
& +\int_{0}^{t}\left(\frac{\partial}{\partial \mathbf{y}} \varphi\right)\left(s, W_{s}, e^{t A} \xi\right) \mathrm{d} W_{s}+\frac{1}{2} \sum_{i=1}^{k} \int_{0}^{t}\left(\frac{\partial^{2}}{\partial y_{i}^{2}} \varphi\right)\left(s, W_{s}, e^{t A} \xi\right) \mathrm{d} s \\
= & e^{t A} \xi-\int_{0}^{t} \frac{1}{2} \sum_{i=1}^{k}\left(L_{i}\right)^{2} \varphi\left(s, W_{s}, e^{t A} \xi\right) \mathrm{d} s+\int_{0}^{t} e^{(t-s) A} B\left(X_{s}\right) \mathrm{d} W_{s} \\
& +\frac{1}{2} \sum_{i=1}^{k} \int_{0}^{t}\left(L_{i}\right)^{2} \varphi\left(s, W_{s}, e^{t A} \xi\right) \mathrm{d} s=e^{t A} \xi+\int_{0}^{t} e^{(t-s) A} B\left(X_{s}\right) \mathrm{d} W_{s} .
\end{aligned}
$$

Combining this and (102) completes the proof of Proposition 3.3 .

\subsection{Stochastic heat equations with nonlinear multiplicative noise}

Proposition 3.4. Assume the setting in Subsection 3.1, let $r, \beta \in[0, \infty), w \in H_{-\beta} \backslash\{0\}$, $\xi \in \mathcal{M}\left(\mathcal{F}_{0}, \mathcal{B}\left(H_{\delta}\right)\right), B \in \mathcal{C}\left(H, H S\left(\mathbb{R}, H_{-\beta}\right)\right)$ satisfy for all $v \in H, u \in \mathbb{R}$ that $B(v) u=$ $u\|v\|_{H} w$, let $W:[0, T] \times \Omega \rightarrow \mathbb{R}$ be a standard $\left(\mathcal{F}_{t}\right)_{t \in[0, T]}$-Brownian motion, and let $X:[0, T] \times$ 
$\Omega \rightarrow H_{\delta}$ be an $\left(\mathcal{F}_{t}\right)_{t \in[0, T] \text {-predictable stochastic process which fulfills for all } t \in(0, T] \text { that }}$ $X((0, T] \times \Omega) \subseteq H$, that $\mathbb{E}\left[\left\|e^{t A} \xi\right\|_{H_{-r}}^{2}\right]+\int_{0}^{t} \mathbb{E}\left[\left\|e^{(t-s) A} B\left(X_{s}\right)\right\|_{H S\left(\mathbb{R}, H_{-r}\right)}^{2}\right] d s<\infty$, and $\mathbb{P}$-a.s. that $X_{t}=e^{t A} \xi+\int_{0}^{t} e^{(t-s) A} B\left(X_{s}\right) d W_{s}$. Then for all $t \in(0, T]$ it holds that $\mathbb{P}\left(\xi \in H_{-1 / 2}\right)=1$ and

$$
2^{-1 / 2} e^{-|\eta| t}\left(1-e^{-2\left[\eta-\sup \left(\sigma_{p}(A)\right)\right] t}\right)^{1 / 2}\left\|e^{t A} w\right\|_{H_{-r}}\|\xi\|_{L^{2}\left(\mathbb{P} ; H_{-1 / 2}\right)} \leq\left\|X_{t}\right\|_{L^{2}\left(\mathbb{P} ; H_{-r}\right)}<\infty .
$$

Proof. Throughout this proof let $\mathbb{B} \subseteq H$ be an orthonormal basis of $H$ and let $\lambda: \mathbb{B} \rightarrow \mathbb{R}$ be a mapping which satisfies $\sup _{b \in \mathbb{B}}-\lambda_{b}<\eta$, which satisfies $D(A)=\left\{v \in H: \sum_{b \in \mathbb{B}}\left|\lambda_{b}\langle b, v\rangle_{H}\right|^{2}<\right.$ $\infty\}$, and which satisfies for all $v \in D(A)$ that $A v=-\sum_{b \in \mathbb{B}} \lambda_{b}\langle b, v\rangle_{H} b$. Note that for all $t \in(0, T]$ it holds $\mathbb{P}$-a.s. that

$$
\begin{aligned}
\left\|X_{t}\right\|_{H_{-r}}^{2}= & \left\|e^{t A} \xi\right\|_{H_{-r}}^{2}+2\left\langle e^{t A} \xi, \int_{0}^{t} e^{(t-s) A} B\left(X_{s}\right) d W_{s}\right\rangle_{H_{-r}} \\
& +\left\|\int_{0}^{t} e^{(t-s) A} B\left(X_{s}\right) d W_{s}\right\|_{H_{-r}}^{2} .
\end{aligned}
$$

Equation (105) together with Itô's isometry and the assumption that $\forall t \in[0, T]: \mathbb{E}\left[\left\|e^{t A} \xi\right\|_{H_{-r}}^{2}\right]+$ $\int_{0}^{t} \mathbb{E}\left[\left\|e^{(t-s) A} B\left(X_{s}\right)\right\|_{H S\left(\mathbb{R}, H_{-r}\right)}^{2}\right] d s<\infty$ hence prove that for all $t \in(0, T]$ it holds that

$$
\begin{aligned}
\mathbb{E}[ & \left.\left\|X_{t}\right\|_{H_{-r}}^{2}\right]=\mathbb{E}\left[\left\|e^{t A} \xi\right\|_{H_{-r}}^{2}\right]+2 \mathbb{E}\left[\left\langle e^{t A} \xi, \int_{0}^{t} e^{(t-s) A} B\left(X_{s}\right) d W_{s}\right\rangle_{H_{-r}}\right] \\
& +\mathbb{E}\left[\left\|\int_{0}^{t} e^{(t-s) A} B\left(X_{s}\right) d W_{s}\right\|_{H_{-r}}^{2}\right] \\
= & \mathbb{E}\left[\left\|e^{t A} \xi\right\|_{H_{-r}}^{2}\right]+2 \mathbb{E}\left[\left\langle e^{t A} \xi, \mathbb{E}\left[\int_{0}^{t} e^{(t-s) A} B\left(X_{s}\right) d W_{s} \mid \mathcal{F}_{0}\right]\right\rangle_{H_{-r}}\right] \\
& +\int_{0}^{t} \mathbb{E}\left[\left\|e^{(t-s) A} B\left(X_{s}\right)\right\|_{H S\left(\mathbb{R}, H_{-r}\right)}^{2}\right] d s \\
= & \mathbb{E}\left[\left\|e^{t A} \xi\right\|_{H_{-r}}^{2}\right]+\int_{0}^{t} \mathbb{E}\left[\left\|e^{(t-s) A} B\left(X_{s}\right)\right\|_{H S\left(\mathbb{R}, H_{-r}\right)}^{2}\right] d s<\infty .
\end{aligned}
$$


Next we note that for all $t \in(0, T], s \in(0, t)$ it holds $\mathbb{P}$-a.s. that

$$
\begin{aligned}
& \left\|e^{(t-s) A} B\left(X_{s}\right)\right\|_{H S\left(\mathbb{R}, H_{-r}\right)}^{2}=\left\|e^{(t-s) A} B\left(e^{s A} \xi+\int_{0}^{s} e^{(s-u) A} B\left(X_{u}\right) d W_{u}\right)\right\|_{H S\left(\mathbb{R}, H_{-r}\right)}^{2} \\
= & \left\|e^{s A} \xi+\int_{0}^{s} e^{(s-u) A} B\left(X_{u}\right) d W_{u}\right\|_{H}^{2}\left\|e^{(t-s) A} w\right\|_{H_{-r}}^{2} \\
= & \left\|e^{(t-s) A} B\left(e^{s A} \xi\right)\right\|_{H S\left(\mathbb{R}, H_{-r}\right)}^{2}+\left\|e^{(t-s) A} B\left(\int_{0}^{s} e^{(s-u) A} B\left(X_{u}\right) d W_{u}\right)\right\|_{H S\left(\mathbb{R}, H_{-r}\right)}^{2} \\
& +2\left\|e^{(t-s) A} w\right\|_{H_{-r}}^{2}\left\langle e^{s A} \xi, \int_{0}^{s} e^{(s-u) A} B\left(X_{u}\right) d W_{u}\right\rangle_{H} \\
\geq & \left\|e^{(t-s) A} B\left(e^{s A} \xi\right)\right\|_{H S\left(\mathbb{R}, H_{-r}\right)}^{2}+2\left\|e^{(t-s) A} w\right\|_{H_{-r}}^{2}\left\langle e^{s A} \xi, \int_{0}^{s} e^{(s-u) A} B\left(X_{u}\right) d W_{u}\right\rangle_{H} \\
= & \left\|e^{(t-s) A} B\left(e^{s A} \xi\right)\right\|_{H S\left(\mathbb{R}, H_{-r}\right)}^{2} \\
& +2\left\|e^{(t-s) A} w\right\|_{H_{-r}}^{2}\left\langle(\eta-A)^{r} e^{s A} \xi, \int_{0}^{s}(\eta-A)^{-r} e^{(s-u) A} B\left(X_{u}\right) d W_{u}\right\rangle_{H} .
\end{aligned}
$$

In addition, the assumption that $\forall t \in(0, T]: \mathbb{E}\left[\left\|e^{t A} \xi\right\|_{H_{-r}}^{2}\right]<\infty$ implies that for all $t \in(0, T]$ it holds that

$$
\mathbb{E}\left[\left\|e^{t A} \xi\right\|_{H_{r}}^{2}\right] \leq\left\|e^{\frac{t}{2} A}\right\|_{L_{\left(H_{-r}, H_{r}\right)}^{2}}^{2}\left[\left\|e^{\frac{t}{2} A} \xi\right\|_{H_{-r}}^{2}\right]<\infty .
$$

Itô's isometry and the assumption that $\forall t \in(0, T]: \int_{0}^{t} \mathbb{E}\left[\left\|e^{(t-s) A} B\left(X_{s}\right)\right\|_{H S\left(\mathbb{R}, H_{-r}\right)}^{2}\right] d s<\infty$ hence prove that for all $t \in(0, T], s \in(0, t)$ it holds that

$$
\begin{aligned}
\mathbb{E} & {\left[\left\|e^{(t-s) A} B\left(X_{s}\right)\right\|_{H S\left(\mathbb{R}, H_{-r}\right)}^{2}\right] \geq \mathbb{E}\left[\left\|e^{(t-s) A} B\left(e^{s A} \xi\right)\right\|_{H S\left(\mathbb{R}, H_{-r}\right)}^{2}\right] } \\
& +2\left\|e^{(t-s) A} w\right\|_{H_{-r}}^{2} \mathbb{E}\left[\left\langle(\eta-A)^{r} e^{s A} \xi, \int_{0}^{s}(\eta-A)^{-r} e^{(s-u) A} B\left(X_{u}\right) d W_{u}\right\rangle_{H}\right] \\
= & \mathbb{E}\left[\left\|e^{(t-s) A} B\left(e^{s A} \xi\right)\right\|_{H S\left(\mathbb{R}, H_{-r}\right)}^{2}\right] \\
& +2\left\|e^{(t-s) A} w\right\|_{H_{-r}}^{2} \mathbb{E}\left[\left\langle(\eta-A)^{r} e^{s A} \xi, \mathbb{E}\left[\int_{0}^{s}(\eta-A)^{-r} e^{(s-u) A} B\left(X_{u}\right) d W_{u} \mid \mathcal{F}_{0}\right]\right\rangle_{H}\right] \\
= & \mathbb{E}\left[\left\|e^{(t-s) A} B\left(e^{s A} \xi\right)\right\|_{H S\left(\mathbb{R}, H_{-r}\right)}^{2}\right] .
\end{aligned}
$$

Furthermore, we observe that for all $t \in(0, T], s \in(0, t)$ it holds that

$$
\left\|e^{t A} w\right\|_{H_{-r}} \leq\left\|e^{s A}\right\|_{L(H)}\left\|e^{(t-s) A} w\right\|_{H_{-r}} \leq e^{\eta s}\left\|e^{(t-s) A} w\right\|_{H_{-r}} \leq e^{\max \{\eta, 0\} t}\left\|e^{(t-s) A} w\right\|_{H_{-r}} .
$$


Combining (109) with (106) and (110) ensures that for all $t \in(0, T]$ it holds that

$$
\begin{aligned}
& \infty>\mathbb{E}\left[\left\|X_{t}\right\|_{H_{-r}}^{2}\right] \geq \mathbb{E}\left[\left\|e^{t A} \xi\right\|_{H_{-r}}^{2}\right]+\int_{0}^{t} \mathbb{E}\left[\left\|e^{(t-s) A} B\left(e^{s A} \xi\right)\right\|_{H S\left(\mathbb{R}, H_{-r}\right)}^{2}\right] d s \\
& \geq \int_{0}^{t} \mathbb{E}\left[\left\|e^{(t-s) A} B\left(e^{s A} \xi\right)\right\|_{H S\left(\mathbb{R}, H_{-r}\right)}^{2}\right] d s=\int_{0}^{t}\left\|e^{(t-s) A} w\right\|_{H_{-r}}^{2} \mathbb{E}\left[\left\|e^{s A} \xi\right\|_{H}^{2}\right] d s \\
& \geq e^{-2 \max \{\eta, 0\} t}\left\|e^{t A} w\right\|_{H_{-r}}^{2} \int_{0}^{t} \mathbb{E}\left[\left\|e^{s A} \xi\right\|_{H}^{2}\right] d s \\
& =e^{-2 \max \{\eta, 0\} t}\left\|e^{t A} w\right\|_{H_{-r}}^{2} \sum_{b \in \mathbb{B}} \int_{0}^{t} \mathbb{E}\left[\left|\left\langle e^{s A} b, \xi\right\rangle_{H}\right|^{2}\right] d s \\
& =e^{-2 \max \{\eta, 0\} t}\left\|e^{t A} w\right\|_{H_{-r}}^{2} \sum_{b \in \mathbb{B}} \int_{0}^{t} e^{-2\left(\lambda_{b}+\eta\right) s} e^{2 \eta s} \mathbb{E}\left[\left|\langle b, \xi\rangle_{H}\right|^{2}\right] d s \\
& \geq e^{-2|\eta| t}\left\|e^{t A} w\right\|_{H_{-r}}^{2} \sum_{b \in \mathbb{B}} \int_{0}^{t} e^{-2\left(\lambda_{b}+\eta\right) s} \mathbb{E}\left[\left|\langle b, \xi\rangle_{H}\right|^{2}\right] d s \\
& =e^{-2|\eta| t}\left\|e^{t A} w\right\|_{H_{-r}}^{2} \sum_{b \in \mathbb{B}} \frac{\left(1-e^{-2\left(\lambda_{b}+\eta\right) t}\right) \mathbb{E}\left[\left|\langle b, \xi\rangle_{H}\right|^{2}\right]}{2\left(\lambda_{b}+\eta\right)} \\
& =\frac{\left\|e^{t A} w\right\|_{H_{-r}}^{2} \sum_{b \in \mathbb{B}}\left(1-e^{-2\left(\lambda_{b}+\eta\right) t}\right) \mathbb{E}\left[\left|\left\langle(\eta-A)^{-1 / 2} b, \xi\right\rangle_{H}\right|^{2}\right]}{2 e^{2|\eta| t}} \\
& \geq \frac{\left(1-e^{-2\left(\inf _{b \in \mathbb{B}} \lambda_{b}+\eta\right) t}\right)\left\|e^{t A} w\right\|_{H_{-r}}^{2}}{2|\eta| t} \mathbb{E}\left[\left|\left\langle(\eta-A)^{-1 / 2} b, \xi\right\rangle_{H}\right|^{2}\right]
\end{aligned}
$$

This and the assumption that $w \neq 0$, in particular, assure that $\mathbb{E}\left[\sum_{b \in \mathbb{B}}\left|\left\langle(\eta-A)^{-1 / 2} b, \xi\right\rangle_{H}\right|^{2}\right]<$ $\infty$. Hence, we obtain that $\mathbb{P}\left(\xi \in H_{-1 / 2}\right)=1$. This and (111) complete the proof of Proposition 3.4 .

\subsection{Nonlinear heat equations}

Proposition 3.5. Let $\left(H,\|\cdot\|_{H},\langle\cdot, \cdot\rangle_{H}\right)$ be a separable $\mathbb{R}$-Hilbert space with $\#_{H}(H)>1$, let $\mathbb{B} \subseteq H$ be an orthonormal basis of $H$, let $(\Omega, \mathcal{F}, \mathbb{P})$ be a probability space, let $T \in(0, \infty)$, $\eta, \delta \in \mathbb{R}$, let $\lambda: \mathbb{B} \rightarrow \mathbb{R}$ be a function which satisfies $\sup _{b \in \mathbb{B}}\left(-\lambda_{b}\right)<\eta$, let $A: D(A) \subseteq H \rightarrow$ $H$ be a linear operator which satisfies $D(A)=\left\{v \in H: \sum_{b \in \mathbb{B}}\left|\lambda_{b}\langle b, v\rangle_{H}\right|^{2}<\infty\right\}$ and which satisfies for all $v \in D(A)$ that $A v=-\sum_{b \in \mathbb{B}} \lambda_{b}\langle b, v\rangle_{H} b$, let $\left(H_{r},\|\cdot\|_{H_{r}},\langle\cdot, \cdot\rangle_{H_{r}}\right), r \in \mathbb{R}$, be a family of interpolation spaces associated to $\eta-A$, let $w \in H, b_{0} \in \mathbb{B}, \xi \in \mathcal{M}\left(\mathcal{F}, \mathcal{B}\left(H_{\delta}\right)\right)$, $F \in \mathcal{C}(H, H)$ satisfy for all $v \in H$ that $\left\langle b_{0}, w\right\rangle_{H}>0, w=\left\langle b_{0}, w\right\rangle_{H} b_{0}$, and $F(v)=\|v\|_{H} w$, and let $X \in \mathcal{M}\left(\mathcal{B}([0, T]) \otimes \mathcal{F}, \mathcal{B}\left(H_{\delta}\right)\right)$ satisfy for all $t \in(0, T]$ that $X((0, T] \times \Omega) \subseteq H$, $\mathbb{P}$-a.s. that $\int_{0}^{t}\left\|e^{(t-s) A} F\left(X_{s}\right)\right\|_{H_{\delta}} d s<\infty$, and $\mathbb{P}$-a.s. that $X_{t}=e^{t A} \xi+\int_{0}^{t} e^{(t-s) A} F\left(X_{s}\right) d s$. Then for all 
$t \in(0, T]$ it holds that $\mathbb{P}\left(\xi \in H_{-1}\right)=1$ and $\mathbb{P}$-a.s. that

$$
\begin{aligned}
& \left\langle b_{0}, w\right\rangle_{H} e^{-\left(\lambda_{b_{0}}+|\eta|\right) t}\left[1-e^{-\left(\inf _{b \in \mathbb{B}} \lambda_{b}+\eta\right) t}\right]\left\|\xi-\left\langle b_{0}, \xi\right\rangle_{H} b_{0}\right\|_{H_{-1}} \\
& \leq\left\langle b_{0}, X_{t}-e^{t A} \xi\right\rangle_{H} \leq\left\|X_{t}-e^{t A} \xi\right\|_{H}<\infty .
\end{aligned}
$$

Proof. Throughout this proof let $P \in L\left(H_{\min \{\delta, 0\}}\right)$ be the linear operator with the property that for all $v \in H$ it holds that $P(v)=v-\left\langle b_{0}, v\right\rangle_{H} b_{0}$. We observe that the assumption that $X((0, T] \times \Omega) \subseteq H$ implies that for all $t \in(0, T]$ it holds $\mathbb{P}$-a.s. that

$$
\begin{aligned}
& \infty>\left\|X_{t}-e^{t A} \xi\right\|_{H}=\left\langle b_{0}, X_{t}-e^{t A} \xi\right\rangle_{H}=\int_{0}^{t}\left\langle b_{0}, e^{(t-s) A} F\left(X_{s}\right)\right\rangle_{H} d s \\
& =\int_{0}^{t}\left\langle b_{0}, e^{(t-s) A} w\right\rangle_{H}\left\|X_{s}\right\|_{H} d s=\int_{0}^{t}\left\langle b_{0}, w\right\rangle_{H} e^{-\left(\lambda_{b_{0}}+\eta\right)(t-s)} e^{\eta(t-s)}\left\|X_{s}\right\|_{H} d s \\
& \geq\left\langle b_{0}, w\right\rangle_{H} e^{\min \{\eta, 0\} t} \int_{0}^{t} e^{-\left(\lambda_{b_{0}}+\eta\right)(t-s)}\left\|P X_{s}\right\|_{H} d s \\
& =\left\langle b_{0}, w\right\rangle_{H} e^{\min \{\eta, 0\} t} \int_{0}^{t} e^{-\left(\lambda_{b_{0}}+\eta\right)(t-s)}\left\|e^{s A} P \xi\right\|_{H} d s \\
& \geq\left\langle b_{0}, w\right\rangle_{H} e^{-\left(\lambda_{b_{0}}+\max \{\eta, 0\}\right) t} \int_{0}^{t}\left\|e^{s A} P \xi\right\|_{H} d s \\
& =\left\langle b_{0}, w\right\rangle_{H} e^{-\left(\lambda_{b_{0}}+\max \{\eta, 0\}\right) t} \int_{0}^{t}\left[\sum_{b \in \mathbb{B} \backslash\left\{b_{0}\right\}}\left|e^{-\left(\lambda_{b}+\eta\right) s} e^{\eta s}\langle b, \xi\rangle_{H}\right|^{2}\right]^{1 / 2} d s .
\end{aligned}
$$

This and the Minkowski integral inequality imply that for all $t \in(0, T]$ it holds $\mathbb{P}$-a.s. that

$$
\begin{aligned}
& \infty>\left\|X_{t}-e^{t A} \xi\right\|_{H} \geq\left\langle b_{0}, X_{t}-e^{t A} \xi\right\rangle_{H} \\
& \geq\left\langle b_{0}, w\right\rangle_{H} e^{-\left(\lambda_{b_{0}}+|\eta|\right) t}\left[\sum_{b \in \mathbb{B} \backslash\left\{b_{0}\right\}}\left|\int_{0}^{t}\right| e^{-\left(\lambda_{b}+\eta\right) s}\langle b, \xi\rangle_{H}|d s|^{2}\right]^{1 / 2} \\
& =\left\langle b_{0}, w\right\rangle_{H} e^{-\left(\lambda_{b_{0}}+|\eta|\right) t}\left[\sum_{b \in \mathbb{B} \backslash\left\{b_{0}\right\}} \frac{\left[1-e^{-\left(\lambda_{b}+\eta\right) t}\right]^{2}\left|\langle b, \xi\rangle_{H}\right|^{2}}{\left|\lambda_{b}+\eta\right|^{2}}\right]^{1 / 2} \\
& \geq\left\langle b_{0}, w\right\rangle_{H} e^{-\left(\lambda_{b_{0}}+|\eta|\right) t}\left[1-e^{-\left(\inf _{b \in \mathbb{B}} \lambda_{b}+\eta\right) t}\right]\left[\sum_{b \in \mathbb{B} \backslash\left\{b_{0}\right\}}\left|\left\langle(\eta-A)^{-1} b, \xi\right\rangle_{H}\right|^{2}\right]^{1 / 2} .
\end{aligned}
$$

The assumption that $\left\langle b_{0}, w\right\rangle_{H}>0$ hence implies that it holds $\mathbb{P}$-a.s. that

$$
\sum_{b \in \mathbb{B}}\left|\left\langle(\eta-A)^{-1} b, \xi\right\rangle_{H}\right|^{2}<\infty .
$$

This ensures that $\mathbb{P}\left(\xi \in H_{-1}\right)=1$. This together with (114) completes the proof of Proposition 3.5 .

\section{Acknowledgments}

Stig Larsson and Christoph Schwab are gratefully acknowledged for some useful comments. This project has been supported through the SNSF-Research project 200021_156603 "Numerical approximations of nonlinear stochastic ordinary and partial differential equations". 


\section{References}

[1] Andersson, A., And Larsson, S. Weak convergence for a spatial approximation of the nonlinear stochastic heat equation. Math. Comp. 85, 299 (2016), 1335-1358.

[2] BréHIER, C.-E. Approximation of the invariant measure with an Euler scheme for stochastic PDEs driven by space-time white noise. Potential Anal. 40, 1 (2014), 1-40.

[3] BrÉhier, C.-E., AND Kopec, M. Approximation of the invariant law of SPDEs: error analysis using a Poisson equation for a full-discretization scheme. doi:10.1093/imanum/drw030.

[4] Brzeźniak, Z. On stochastic convolution in Banach spaces and applications. Stochastics Stochastics Rep. 61, 3-4 (1997), 245-295.

[5] Brzeźniak, Z., van Neerven, J. M. A. M., Veraar, M. C., and Weis, L. Itô's formula in UMD Banach spaces and regularity of solutions of the Zakai equation. $J$. Differential Equations 245, 1 (2008), 30-58.

[6] Carmona, R. A., and Molchanov, S. A. Parabolic Anderson problem and intermittency. Mem. Amer. Math. Soc. 108, 518 (1994), viii+125.

[7] Chen, L., and Dalang, R. C. Hölder-continuity for the nonlinear stochastic heat equation with rough initial conditions. Stoch. Partial Differ. Equ. Anal. Comput. 2, 3 (2014), 316-352.

[8] Chen, L., And Dalang, R. C. Moments and growth indices for the nonlinear stochastic heat equation with rough initial conditions. Ann. Probab. 43, 6 (2015), 3006-3051.

[9] Conus, D., Jentzen, A., And Kurniawan, R. Weak convergence rates of spectral Galerkin approximations for SPDEs with nonlinear diffusion coefficients. arXiv:1408.1108 (2014), 29 pages.

[10] Da Prato, G., And ZabczyK, J. Stochastic equations in infinite dimensions, vol. 44 of Encyclopedia of Mathematics and its Applications. Cambridge University Press, Cambridge, 1992.

[11] Da Prato, G., And Zabczyk, J. Ergodicity for infinite-dimensional systems, vol. 229 of London Mathematical Society Lecture Note Series. Cambridge University Press, Cambridge, 1996.

[12] Debussche, A. Weak approximation of stochastic partial differential equations: the nonlinear case. Math. Comp. 80, 273 (2011), 89-117.

[13] Gorenflo, R., Kilbas, A. A., Mainardi, F., And Rogosin, S. V. Mittag-Leffler functions, related topics and applications. Springer Monographs in Mathematics. Springer, Heidelberg, 2014. 
[14] HenRy, D. Geometric theory of semilinear parabolic equations, vol. 840 of Lecture Notes in Mathematics. Springer-Verlag, Berlin-New York, 1981.

[15] Hoffmann-Jørgensen, J. Probability with a view toward statistics. Vol. I. Chapman \& Hall Probability Series. Chapman \& Hall, New York, 1994.

[16] Jentzen, A., And Kloeden, P. E. Taylor approximations for stochastic partial differential equations, vol. 83 of CBMS-NSF Regional Conference Series in Applied Mathematics. Society for Industrial and Applied Mathematics (SIAM), Philadelphia, PA, 2011.

[17] Jentzen, A., And Kurniawan, R. Weak convergence rates for Euler-type approximations of semilinear stochastic evolution equations with nonlinear diffusion coefficients. arXiv:1501.03539 (2015), 51 pages.

[18] Jentzen, A., And RöCKner, M. Regularity analysis for stochastic partial differential equations with nonlinear multiplicative trace class noise. J. Differential Equations 252, 1 (2012), 114-136.

[19] Klenke, A. Probability theory. Universitext. Springer-Verlag London Ltd., London, 2008. A comprehensive course, Translated from the 2006 German original.

[20] Kopec, M. Quelques contributions à l'analyse numérique d'équations stochastiques. $P h D$ thesis, ENS Rennes (2014), viii+189.

[21] Kruse, R., And Larsson, S. Optimal regularity for semilinear stochastic partial differential equations with multiplicative noise. Electron. J. Probab. 17 (2012), no. 65, 19.

[22] Parthasarathy, K. R. Probability measures on metric spaces. Probability and Mathematical Statistics, No. 3. Academic Press, Inc., New York-London, 1967.

[23] PRÉvôt, C., AND RöCKNer, M. A concise course on stochastic partial differential equations, vol. 1905 of Lecture Notes in Mathematics. Springer, Berlin, 2007.

[24] Renardy, M., And Rogers, R. C. An introduction to partial differential equations, vol. 13 of Texts in Applied Mathematics. Springer-Verlag, New York, 1993.

[25] Rudin, W. Principles of mathematical analysis, third ed. McGraw-Hill Book Co., New York-Auckland-Düsseldorf, 1976. International Series in Pure and Applied Mathematics.

[26] Sell, G. R., And You, Y. Dynamics of evolutionary equations, vol. 143 of Applied Mathematical Sciences. Springer-Verlag, New York, 2002.

[27] van Neerven, J., Veraar, M., And Weis, L. Maximal $L^{p}$-regularity for stochastic evolution equations. SIAM J. Math. Anal. 44, 3 (2012), 1372-1414.

[28] van Neerven, J. M. A. M., Veraar, M. C., And Weis, L. Stochastic evolution equations in UMD Banach spaces. J. Funct. Anal. 255, 4 (2008), 940-993. 
[29] WAng, X. Weak error estimates of the exponential Euler scheme for semi-linear SPDEs without Malliavin calculus. Discrete Contin. Dyn. Syst. 36, 1 (2016), 481-497.

[30] WAnG, X., And Gan, S. Weak convergence analysis of the linear implicit Euler method for semilinear stochastic partial differential equations with additive noise. J. Math. Anal. Appl. 398, 1 (2013), 151-169. 